- Title: Development of a EUV test facility at the Marshall Space Flight Center

Authors: Edward West ${ }^{1}$, Steve Pavelitz ${ }^{1}$, Ken Kobayashi ${ }^{2}$, Brian Robinson ${ }^{2}$, Jonathan Cirtain ${ }^{1}$, Jessica Gaskin ${ }^{1}$, and Amy Winebarger ${ }^{1}$

${ }_{1}^{1}$ NASA/Marshall Space Flight Center, Huntsville, Al, 35805

2 University of Alabama in Huntsville, Huntsville, Al, 35805

- Abstract:

This paper will describe a new EUV test facility that is being developed at the Marshall Space Flight Center (MSFC) to test EUV telescopes. Two flight programs, HiC - high resolution coronal imager (sounding rocket) and SUVI - Solar Ultraviolet Imager (GOES-R), set the requirements for this new facility. This paper will discuss those requirements, the EUV source characteristics, the wavelength resolution that is expected and the vacuum chambers (Stray Light Facility, Xray Calibration Facility and the EUV test chamber) where this facility will be used.

BIO for primary author:

Edward West has been working with the Solar Physics Group at Marshall Space Flight Center (MSFC) since the Skylab missions in 1973. He has worked on several ground-based solar telescopes that measure the Sun's vector magnetic fields and is currently working on a sounding rocket telescope called SUMI, the Solar Ultraviolet Magnetograph Investigation, to measure the magnetic field in the Sun's transition region. 


\title{
Development of an EUV test facility at the Marshall Space Flight Center
}

\author{
Edward West $^{\mathrm{a}}$, Steve Pavelitz ${ }^{\mathrm{a}}$, Ken Kobayashi ${ }^{\mathrm{b}}$, Brian Robinson ${ }^{\mathrm{b}}$, Jonathan Cirtain ${ }^{\mathrm{a}}$, \\ Jessica Gaskin ${ }^{\mathrm{a}}$, Amy Winebarger ${ }^{\mathrm{a}}$ Linda Krause $^{\mathrm{a}}$, Michael McGuirk ${ }^{\mathrm{c}}$ and Jonathan Darnel ${ }^{\mathrm{d}}$ \\ ${ }^{\text {a }}$ Marshall Space Flight Center/NASA, 320 Sparkman Dr., Huntsville, AL 35805-1912 \\ ${ }^{b}$ The University of Alabama in Huntsville, 320 Sparkman Dr., Huntsville, AL 35805-1912 \\ ${ }^{\mathrm{c}}$ MIT-Lincoln Laboratory, 244 Wood Street, Lexington, MA, 02420-9108 \\ ${ }^{d}$ I. M. Systems Group, Rockville, MD 20852
}

\begin{abstract}
This paper will describe a new Extreme Ultraviolet (EUV) test facility that is being developed at the Marshall Space Flight Center (MSFC) to test EUV telescopes. Two flight programs, Hi-C, the high resolution coronal imager (a sounding rocket program), and SUVI, the Solar Ultraviolet Imager (GOES-R), set the requirements for this new facility. This paper will discuss those requirements, the EUV source characteristics, the wavelength resolution that is expected and the vacuum chambers (Stray Light Facility, Xray Calibration Facility and the NSSTC EUV test chamber) where this facility will be used.
\end{abstract}

Keywords: Extreme Ultraviolet, EUV, X-Ray test facilities, Hi-C, SUVI, EUV telescopes, EUV source

\section{INTRODUCTION}

A new Extreme Ultraviolet (EUV) source has been added to the test facilities at the Marshall Space Flight Center (MSFC) to extend the wavelength coverage from Xray to the EUV at the MSFC Xray Calibration Facility (XRCF) and the MSFC Stray Light Facility (SLF). This paper will describe those facilities, which have primarily been used for Xray work (§2), the requirements and goals of missions (SUVI and Hi-C, §3) that required a strong EUV source (9-100nm) at those facilities, the test facility that has been setup at the National Space Science and Technology Center (NSSTC) (§4), the test data that has been acquired at the NSSTC using this new EUV source and the data system to support and monitor its status (§5).

\section{DESCRIPTION OF THE MSFC TEST FACILITIES}

The MSFC EUV development program will provide a strong EUV source, an EUV monochromator for wavelength calibrations and the detectors and data system to support Marshall's major test facilities (XRCF and CLF) for space based telescopes. The section will describe those facilities ( $\$ 2.1$ and \$2.2) and the small vacuum chamber (\$2.3) that has been setup to do the initial measurements on the source and develop the EUV test procedures.

\subsection{X-ray Calibration Facility (XRCF)}

Marshall Space Flight Center's (MSFC's) X-ray Calibration Facility (Figure 1) is a world-class, one-of-a-kind facility that was constructed in 1975. The XRCF is the largest X-ray, optical test site in the world and has a 2,000-square foot, class 10,000 area for unpacking and assembling hardware and a 6,000- square foot, class 2,000 vertical laminar flow clean room. The XRCF's vacuum chamber is a 24 -foot by 75 -foot stainless steel

Table 1. Summary of XRCF capabilities

- $\quad$ Test chamber: $\varnothing 7.3 \mathrm{~m}$ by $22.8 \mathrm{~m}$ long

- $\quad$ Tube: $\varnothing 1.5 \mathrm{~m}$ by $518 \mathrm{~m}$ long

- $\quad$ Source to detector distance: $538 \mathrm{~m}$

- Cryogenic pumps capable of $10^{-7}$ torr

- $\quad$ Test chamber has thermal shrouds for deep space simulations $\left(\sim 35{ }^{\circ} \mathrm{K}\right)$

- $\quad$ Five degree-of-freedom optical mount 


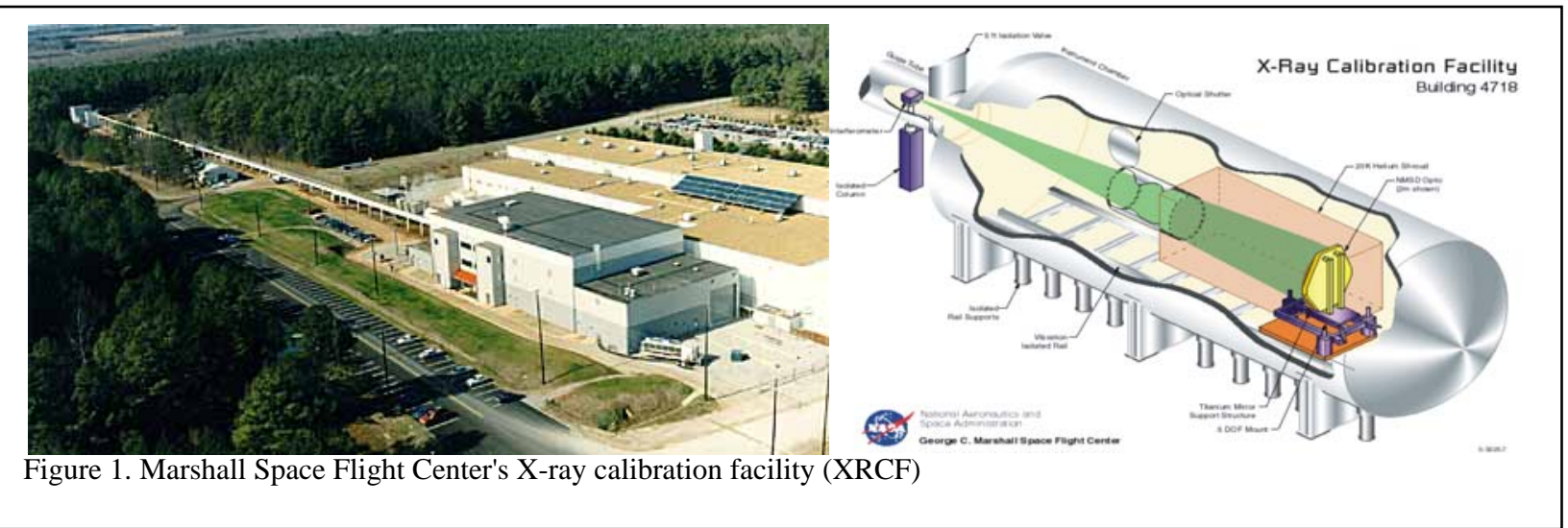

compartment, capable of providing temperatures from $-180^{\circ} \mathrm{F}$ to $+180^{\circ} \mathrm{F}$ and vacuums to $10^{-7}$ Torr.

With a 518 meter long, stainless steel guide tube between the source and the calibration chamber, this facility can provide a "collimated" beam to test space telescopes. The guide tube varies in diameter from 0.9 meters at the source to 1.5 meters at the entrance to the main test chamber. The facility was originally developed in the 1970's for ground testing and calibration of the High Energy Astrophysics Observatories

From 1977 through 1989 the facility tested and calibrated ten sounding rockets with X-ray payloads, a grating spectrometer for the Geosynchronous Earth Orbiting Satellite (GEOS), the Yohkoh soft X-ray telescope (SXT, 19912004), and components developed for the Advanced X-ray Astrophysics Facility (Chandra). In late 1989, the facility was upgraded in size and capability to perform the AXAF end-to-end calibrations (see http://optics.nasa.gov/facilities/xraycal_history.html).

While the facility has been used for other missions, most have supported X-ray programs ${ }^{1,2}$. This program will expand the XRCF wavelength range for EUV missions such as NOAA's GOES-R instrument called SUVI (§3.2).

\subsection{Stray Light Facility (SLF)}

In addition to the 530m X-Ray Calibration Facility, Marshall Space Flight Center operates a 104m long (source-to-detector) Stray Light Facility (SLF, Figure 2). Originally developed for stray-light testing visible-light systems, the SLF now serves as an inexpensive facility to measure the performance of and calibrate X-ray optics and detectors. Our program began with our need to expand this facility's wavelength band into the EUV for our Hi-C sounding rocket program (§3.1).

The available $\mathrm{X}$-ray sources at this facility can span the energy range of 0.2 to $100 \mathrm{keV}$ (6.2nm down to $0.012 \mathrm{~nm}$ ) in support of soft and hard X-ray optics and detectors. To support this testing, the SLF detectors include a frontilluminated CCD (charge-coupled device) and a scanning CZT (cadmiumzinc-telluride) detector, with lowenergy cut-offs of 0.8 and $3 \mathrm{keV}$, respectively ${ }^{2}$.

Several enhancements to the facility

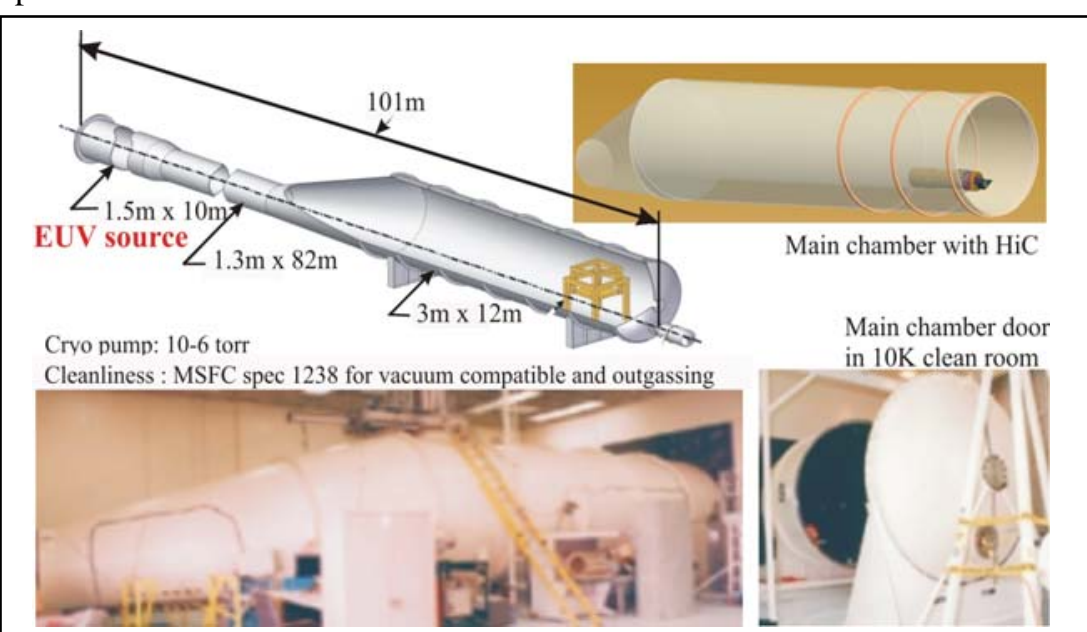

Figure 2. Marshall Space Flight Center's Stray Light facility (SLF)
Table 2. Summary of SLF capabilities

- $\quad$ Test chamber: $\varnothing 3 \mathrm{~m}$ by $12 \mathrm{~m}$ long

- $\quad$ Tube: $\varnothing 1.3 \mathrm{~m}$ by $82 \mathrm{~m}$ long

- $\quad$ Source section: $\varnothing 1.5 \mathrm{~m}$ by $10 \mathrm{~m}$ isolatable

- Cryopumps capable of $10^{-6}$ torr

- $\quad$ Test chamber can accommodate $1 \mathrm{~m}$ optics

- $\quad$ Five degree of freedom optical mount 
were made to support tests of the Constellation-X optics. The major upgrade was the development of a five-degree-offreedom (5-DoF) optical mount and control system to hold and tilt user-provided test equipment such as Hi-C (and possibly SUVI).

Our development program began with the need to expand the SLF wavelength band into the EUV. Originally the required wavelength band was $10-20 \mathrm{~nm}$ for Hi-C (\$3.1) but that was extended to 9-31nm to cover SUVI's wavelength range (§3.2).

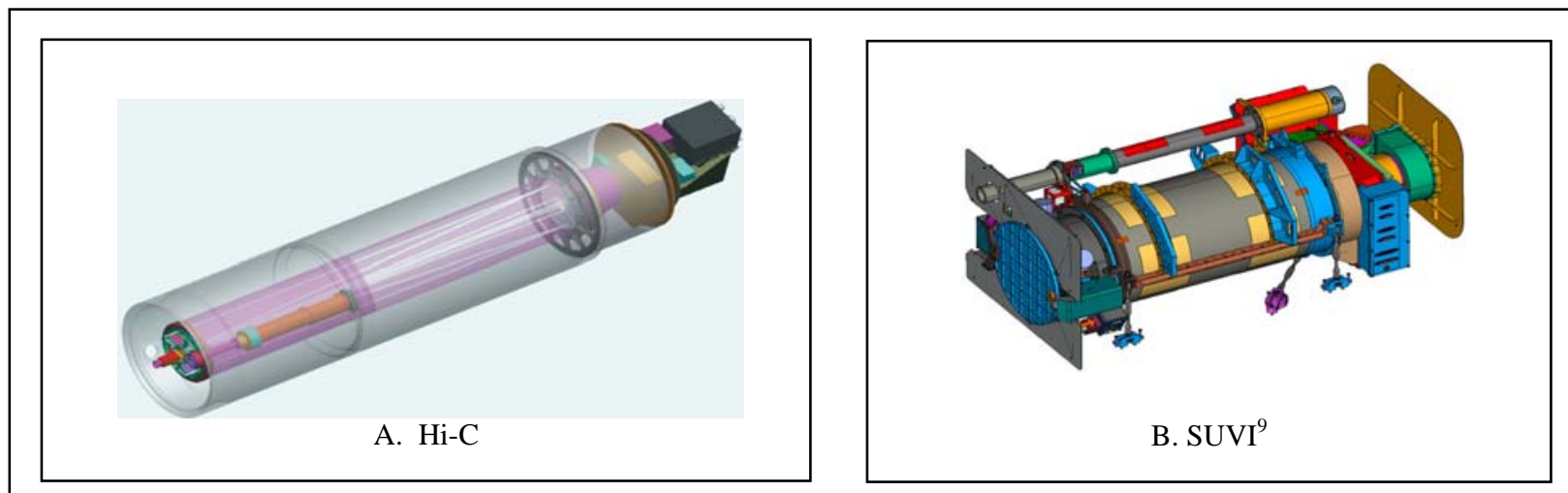

Figure 3. Solar telescopes that will be flown in space that have influence the development of MSFC's EUV facility. $\mathrm{Hi}-\mathrm{C}$ is a MSFC sounding rocket program with a 22cm primary mirror observing a single wavelength, $19.5 \mathrm{~nm}$. SUVI is a solar telescope for NOAA's GOES-R satellite ${ }^{3}$ that has a $20 \mathrm{~cm}$ primary that is segmented into 6 wavelength bands, 9.4, 13.1, 17.1, 19.5, 28.4 and 30.4nm.

\section{MISSIONS THAT INFLUENCED THE EUV SOURCE DEVELOPMENT}

This research effort began with the support of the High Corona imager (Hi-C, sounding rocket program) but most of the source requirements came from a NOAA program called SUVI (GOES-R, Solar Ultraviolet Imager) ${ }^{3}$. This section will provide a brief description of these two programs with emphasis on the requirements that they placed on this development program.

\subsection{High resolution Coronal imager (Hi-C)}

$\mathrm{Hi}-\mathrm{C}$, the High resolution Coronal imager (Figure 3.A), is the first telescope designed to take high spatial resolution $(170 \mathrm{~km})$ images in the extreme ultraviolet. The intent of this sounding rocket program is to demonstrate the technology to acquire images with this resolution and investigate the scientific value of these images. Hi-C is a Ritchey-Chretien telescope with superpolished mirrors that will provide 0.1 arcsec pixels. The mirrors have multilayer coatings that will reflect a single wavelength band at $19.5 \mathrm{~nm}$ to a large format CCD camera.

\subsection{Solar Ultraviolet Imager (SUVI)}

SUVI, the Solar Ultraviolet Imager ${ }^{3,9}$ (Figure 3.B), is a $20 \mathrm{~cm}$ Ritchey-Chretien solar telescope with multilayer coatings on both the primary and secondary. SUVI is being developed for the NOAA GOES-R satellite which will provide full solar disk images at high cadence in the extreme ultraviolet (EUV). Combinations of filters and exposures will allow SUVI to observe coronal holes, $\mathrm{X}$-class flares and estimate temperatures and emission. The purpose of this telescope is to enable NOAA

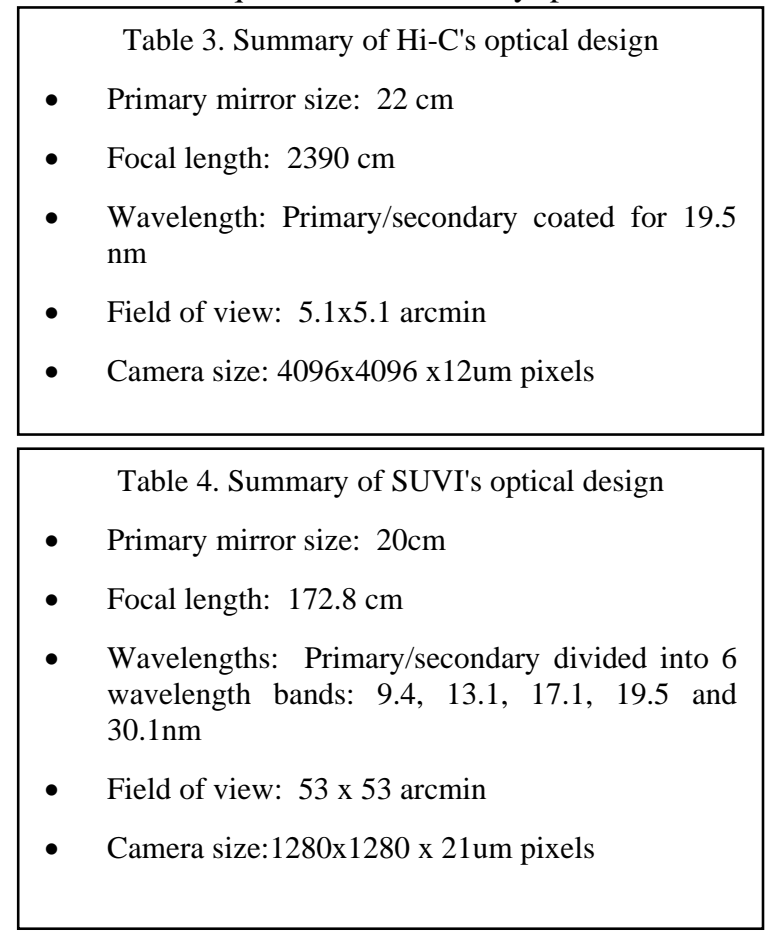


to monitor solar activity and issue real-time alerts.

\subsection{SUVI/Hi-C requirements on EUV source}

The following is a list of the requirements that affected the optical design, the vacuum and mechanical interfaces and the control system for the MSFC EUV source (the flight program that set these requirements in parenthesis):

- To provide adequate flux levels at Hi-C's primary wavelength $(19.5 \mathrm{~nm})$ at the SLF to verify its 0.1 arcsec resolution (Hi-C)

- To provide adequate flux levels at SUVI's six wavelengths bands (9.4, 13.1, 17.1, 19.5, 28.4 and 30.4nm) at the XRCF (see Figure 4) for point source response test (SUVI).

- To monitor and control the source (high voltage and gas flow) to maximize the flux output at each wavelength band that is being tested (SUVI)

- To vary the flux levels of the source (pulse frequency, high voltage and gas flow) for photometric tests (SUVI)

- To produce out-of-band wavelengths for EUV filter response and scattering measurements (SUVI)

- To capture each pulse emitted by the source during the exposure times of the telescope's scientific camera for absolute radiometry calibration (SUVI)

\section{NSSTC EUV TEST FACILITIES}

The section will describe the NSSTC test facilities which consists of: a small vacuum chamber ( $\$ 4.1$ ) to mount our detectors (§4.3) and test optics, the AIXUV source with Luxel thin metal EUV filters (§4.2) to isolate different wavelength bands, a custom EUV transmission grating (§4.4) to monitor the source and a custom EUV monochromator (§4.5, delivery date 7/31/2011) that will be mounted between the online beam of the source and the test facilities (NSSTC, XRCF and SLF) and provide the narrow wavelength bands to test Hi-C and SUVI.

\subsection{EUV vacuum chamber}

While the EUV source will be used at the XRCF and SLF, the scheduling and cost led to the development of a small vacuum chamber $(0.5 \mathrm{~m}$ diameter $\times 1.9 \mathrm{~m}$ long, Figure 5$)$ at the National Space Science and Technology Center (NSSTC) to provide a cost effective facility to test the EUV source, monochromator and its data system, and to develop the control software and procedures that will be used at the XRCF and SLF. Therefore, the EUV data describe in Section 5 were obtained at this facility.

The vacuum systems attached to the chamber are a Varian turbo pump (TV81-M) and a CTI Cyrogenics cyropump (Model 8). While the vacuum system can reach $<10^{-7}$ torr, the typical vacuum levels for the EUV tests in Section 5 are in the $10^{-6}$ to $10^{-5}$ torr range. Since the chamber is short, the absorption loss from any gases in the chamber, typically water, nitrogen

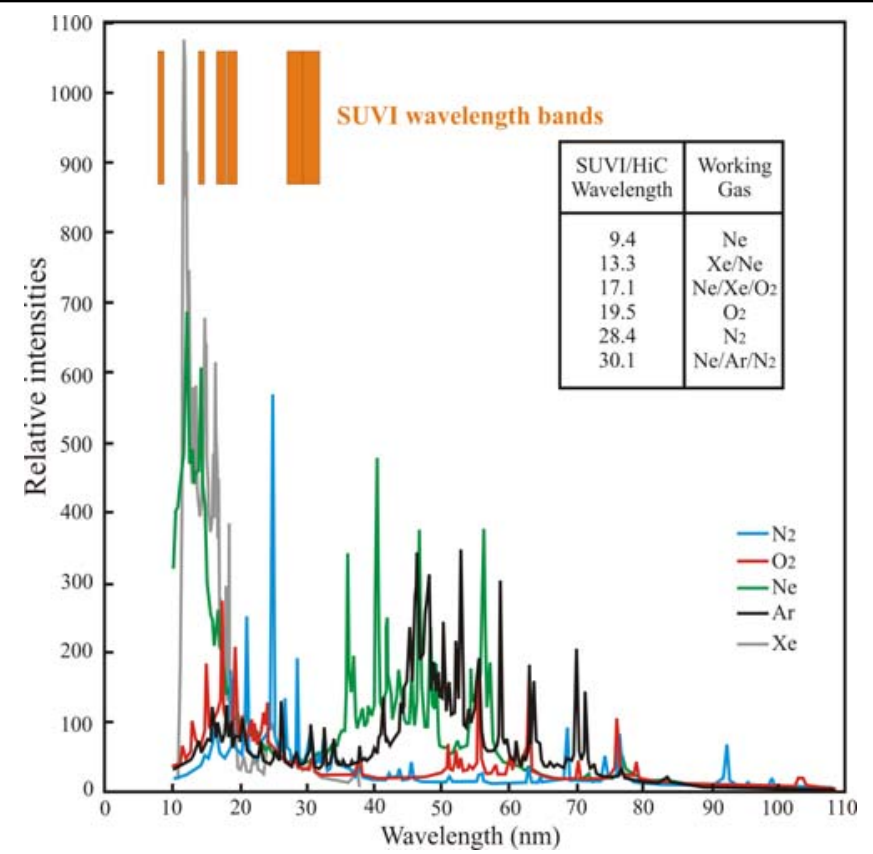

Figure 4. SUVI wavelength bands (orange boxes at top, Hi-C is at $19.5 \mathrm{~nm}$ ) with the working gases that will be used to produce "broadband" emission (BB: Xe, Ne, Ar) and emission lines (EL: N2, O2) in our wavelength range. and oxygen, is small. 
There are two gauges mounted to the test chamber that allow us to monitor the status of our vacuum system: a Granville-Phillips vacuum gauge (VG) and a Amtek residual gas analyzer (RGA). A second set of these gauges is mounted to EUV source beams (Figure $6)$.

The Granville-Phillips gauge is a MicroIon ATM gauge (VG in Figure 6) which combines an ionization gauge with a miniature heat loss sensor and two piezo resistive sensors for a continuous pressure measurement from high vacuum to atmosphere. This gauge has a RS-485 interface so that vacuum information can be stored in the data system monitoring the source.

An Amtek residual gas analyzer (RGA) is used to monitor any contamination or out gassing of the test optics and electronics that have been placed into

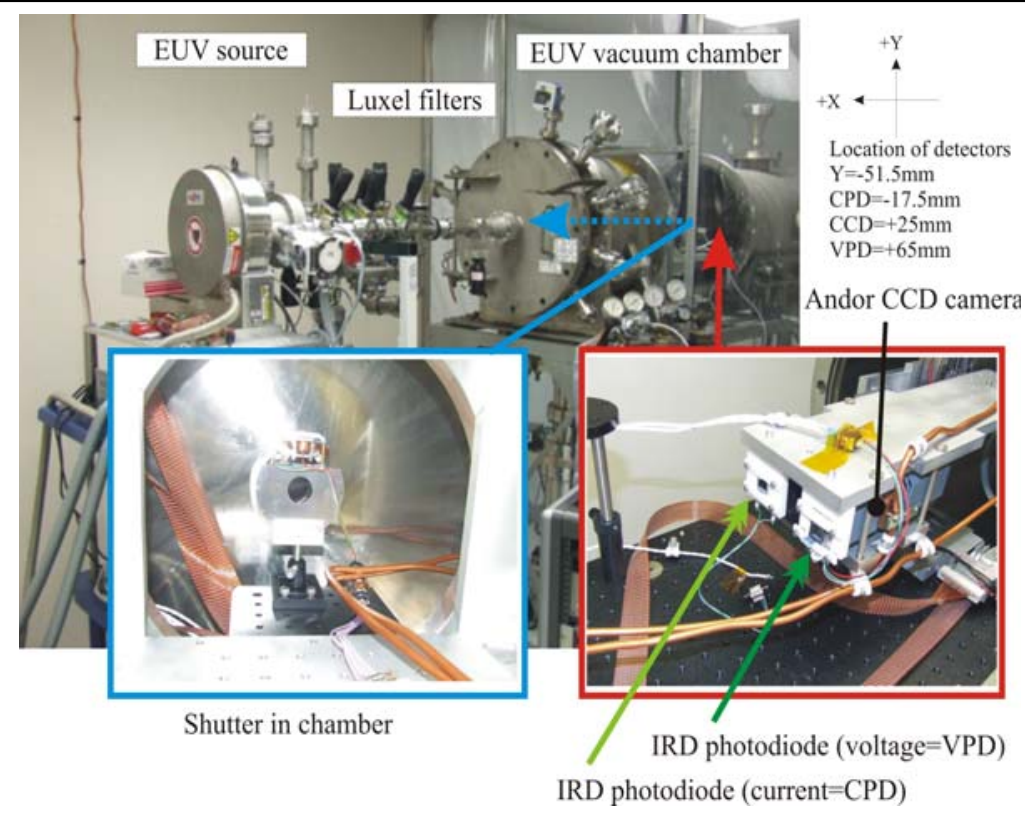

Figure 5. EUV test chamber, source and detector system the test chamber before they are exposed to the EUV source. This RGA (model LC100) is a compact quadrupole mass spectrometer primarily used in the 1-100 AMU range but can also be used as a leak detector (He, Argon) and to monitor any gas from the source that makes it into the test chamber. The RGA is also a backup vacuum gauge. There are two RGAs, one on the test chamber and the second on the monitor beam of the source (Figure 6).

\subsection{EUV source: Online beam - monitor beam(s)}

The manufacturer of our EUV source is AIXUV. The EUV-LAMP-500 consists of two structural components: the discharge head and the control rack.

The discharge head has only those components that are required to be very close to the EUV-source for plasma and vacuum generation. These components are: the discharge body containing capacitor bank, discharge electrodes, the turbo pump and gas flow controllers to control the gas in the lamp, and the pressure gauges and temperature sensors to monitor the status of the lamp ${ }^{3}$. The discharge head has an online beam that is attached to the test chamber and two addition beams that can be used to monitor the source (MB1 and MB2 in Figure 6).

The control rack contains all sub-systems that are needed to allow the user to operate the system and to adjust the high voltage, the gas flow and the discharge frequency for the various gases that we expect to use in the Hi-C and SUVI tests.

Figure 5 shows the AIXUV source attached to the NSSTC vacuum chamber .

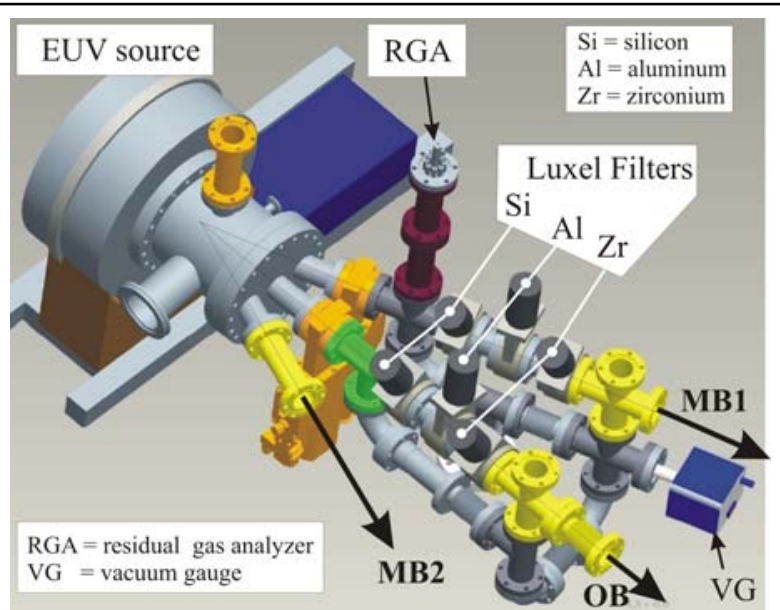

Figure 6. CAD Model of EUV source with location of Luxel EUV filters and vacuum gauges (RGA and VG) 
The source's online beam (OB) and monitor beam (MB1) have three thin Luxel metal filters that can be inserted into the beam to block visible light, to isolate certain wavelength bands and to protect sensitive equipment from contamination by the source. The typical transmission properties of our Luxel filters are shown in Figure 7.

Table 5 lists some of the characteristics of the AIXUV source and the recommended gases for our wavelength bands 5 .

\subsection{Detectors to monitor the source}

Three detectors are being used to characterize the EUV source: two IRD AXUV-100 photodiodes and an Andor DX434-BN X-ray camera.

One of the IRD photodiodes is operated in current mode to capture every EUV pulse created by the source. This

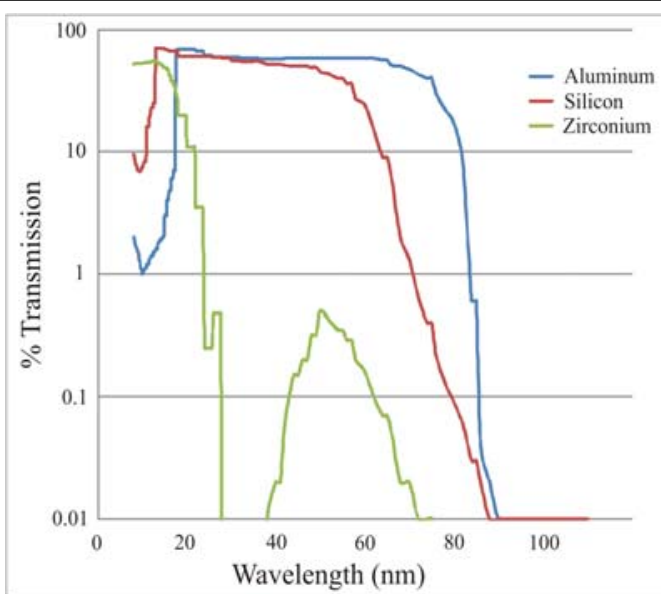

Figure 7. Typical transmission profiles of the three Luxel filters that are attached to the source ${ }^{6}$ (Figure 6). current is then converted into the number of photoelectrons per pulse. Knowing the average wavelength of the EUV source, the number of photons created by the pulse can be estimated. This is important in making absolute radiometry measurements (§5.2). The second IRD photodiode is mounted to an IRD vacuum-compatible amplifier and is used when the signal to noise is low. Since the NSSTC is small compared to the SLF and XRCF, the output of this photodiode has been saturated even with all of the Luxel filters inserted into the beam. We expect that this photodiode will be used when the EUV monochromator arrives and is integrated into the system.

The Andor camera is a 1024x1024 array with13um pixels. This camera is a full frame CCD which requires a shutter to eliminate smearing. Since the camera was bought to make spectral images, vertical binning allows the "smeared" spectral image to be summed into a single wavelength scan which improves the signal to noise $(\mathrm{S} / \mathrm{N})$. Although a shutter can be used (Figure 5), if the wavelength axis is aligned to the readout register of the camera, the shutter is not required. All of the spectral images using the transmission grating (§5.3) were made with this camera.

All of the detectors are currently mounted to linear stages in the EUV vacuum chamber (Figure 5) so that their performance can be documented. This setup will also be used to document the relationship between the monitor beams (MB1 and MB2) and the online beam (OB) before the CCD camera and CPD are moved to their positions: MB1 and MB2 (see Figure 6).

\subsection{Transmission gratings}

While the JY monochromator will be used isolate the wavelength bands required to calibrate SUVI and Hi-C, two transmission gratings will be used in the monitor beams to allow the operator to observe the source's wavelength stability (MB1) in "realtime" and for our radiometry calibrations by observing every pulse created by the source (MB2) during a given exposure time for the scientific camera that is being tested.

The transmission grating that produced the data in Section 5 has a $100 \mathrm{~nm}$ Si3N4 substrate with a 1x1mm patterned area. Within this area, 5000 lines $/ \mathrm{mm}$ are etched into the substrate. Unfortunately, the etching depth was only $~ 60 \mathrm{~nm}$ producing a transmission grating with a Si3N4 "filter". With

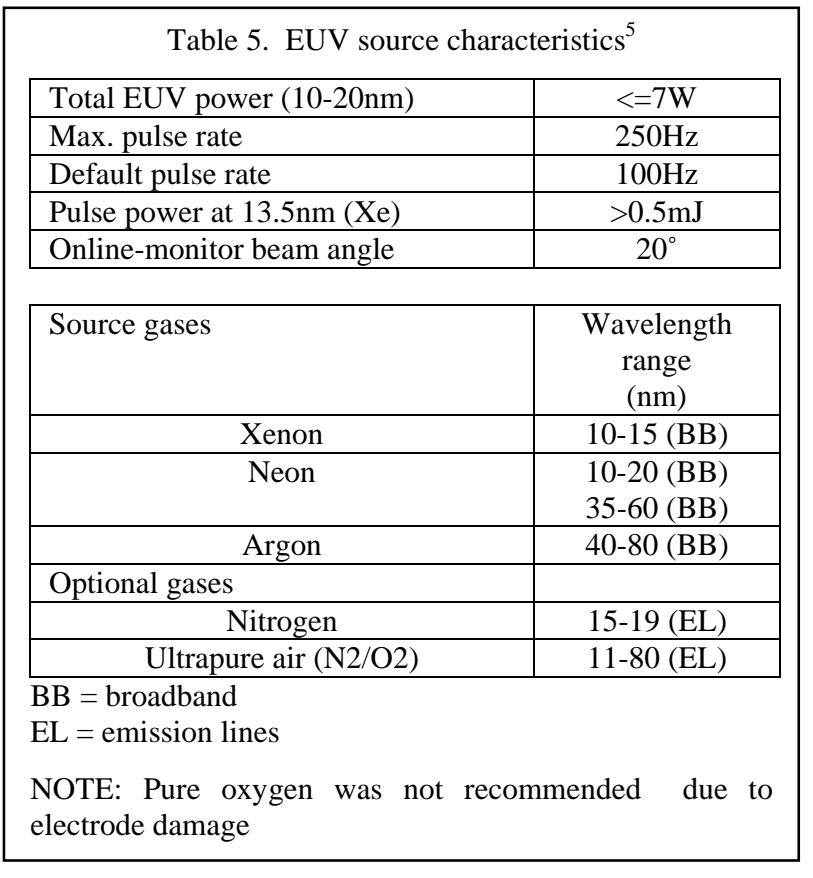




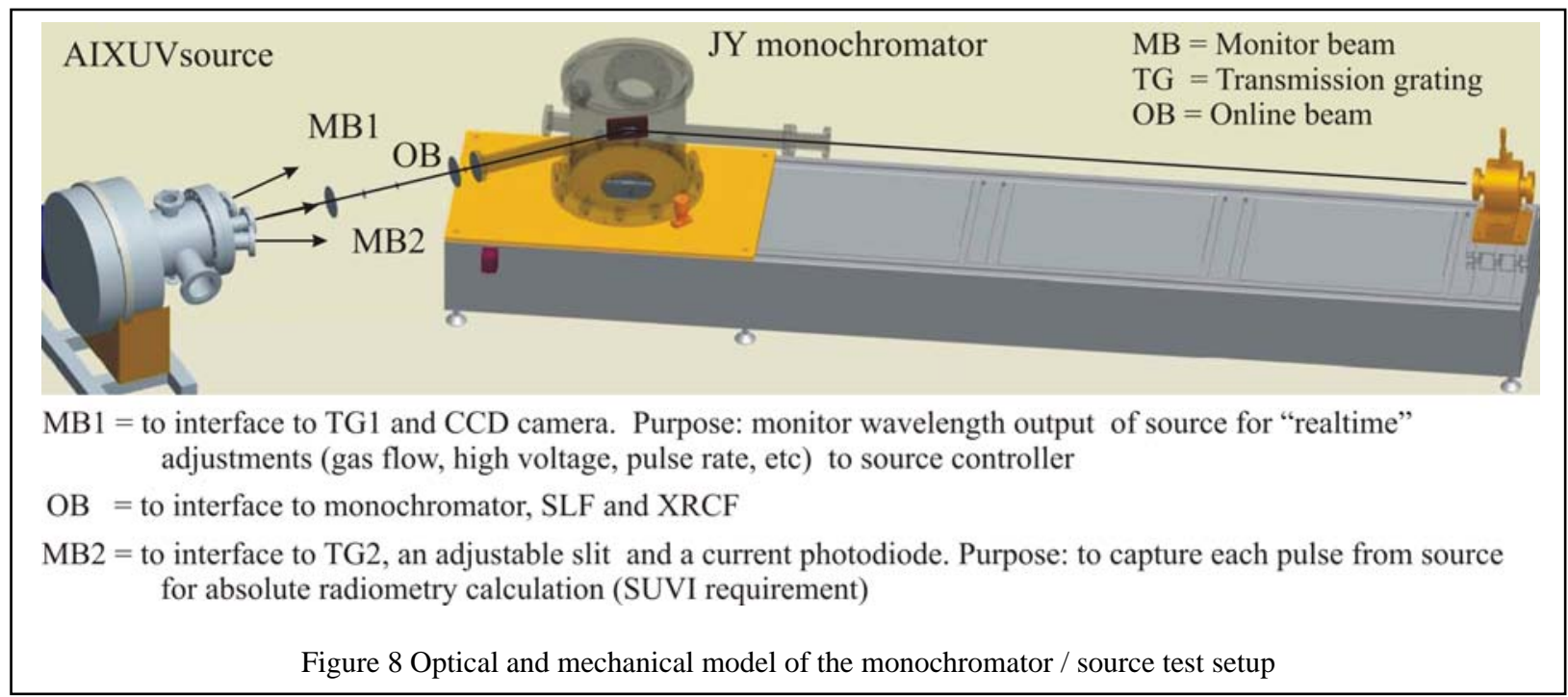

this filter, the Luxel filters were not needed to eliminate the visible light. While Si3N4 has a similar short wavelength cutout as the silicon filter in Figure $7(12.2 \mathrm{~nm})$, the maximum transmission is $29 \%$ at $13 \mathrm{~nm}$ and falls off to less than $0.025 \%$ at $30 \mathrm{~nm}$. This would be an ideal grating for an instrument observing a single wavelength like Hi-C but will not work for a multiline instrument like SUVI. Therefore, to cover all of SUVI's wavelength bands, a new transmission grating with no "substrate filtering" will be required. This will be discussed in greater detail in §5.3.

\subsection{Monochromator interface}

Although the monochromator has not been delivered at the time this paper was written, Figure 8 shows the mechanical and optical model that has been developed for its integration between the source and the NSSTC vacuum chamber this Fall. This instrument is critical to the wavelength calibration of SUVI.

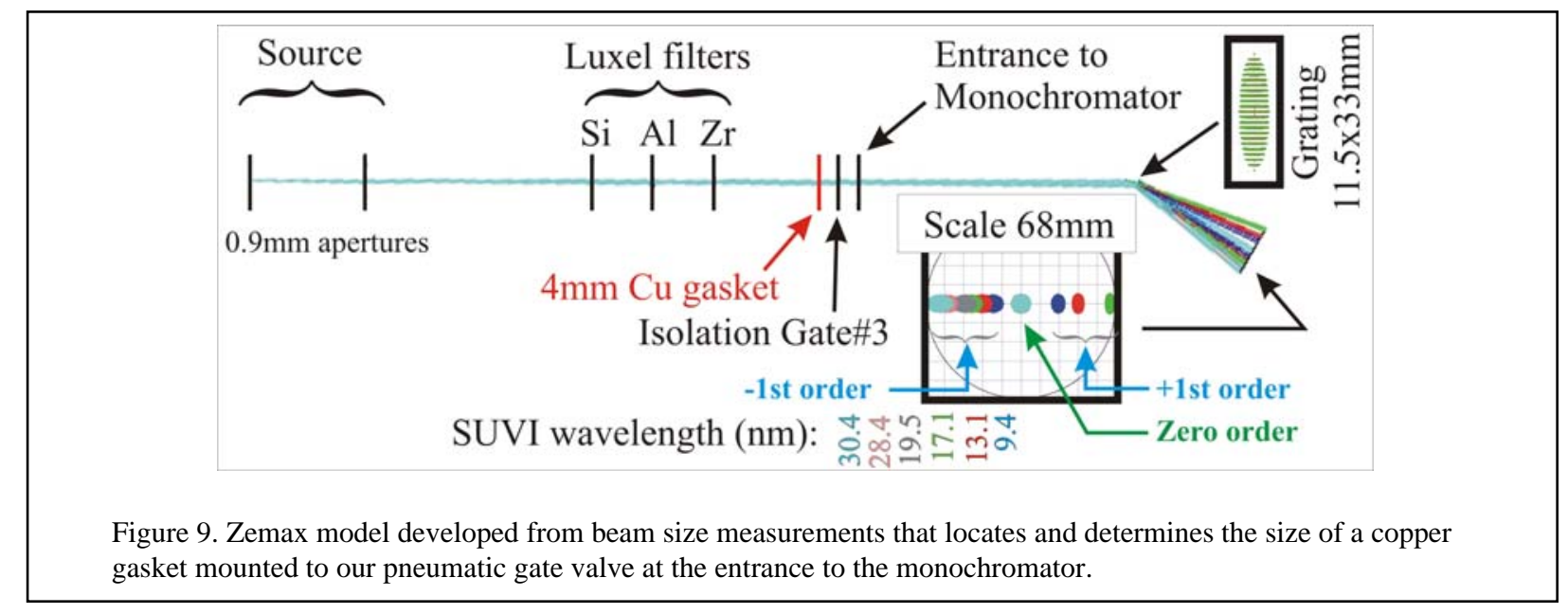

\section{CHARACTERIZING THE EUV SOURCE}

This section will discuss the measurements that have been made on the source: (1) to develop an optical model of the source that could be used to determine the ideal location for a stop in front of the JY monochromator and (2) to determine the "long" term stability of the source (thermal drift, gas flow, etc.) and the short term variation between pulses, With these measurements we calculate the size of the online beam and the integrated photon flux (no wavelength information) that can be expected at the SLF and XRCF. While our transmission gratings are not ideal, we will discuss some of the spectral data they produced from the various gases that will be used with our source and the wavelength resolution that can be expected on this "slit-less" component. 


\subsection{Mapping beam characteristics}

The first step in characterizing our source was to measure the size of the beam at the detectors in the test chamber and develop an optical model to predict the size when a copper stop is placed at different locations along the optical path of the online beam. With this model, the size and location of a copper stop that would reduce the size of the beam at the grating ( $\sim 90 \%$ of its aperture) could be determined (Figure 9). Assuming the Luxel filters are not inserted into the beam to maximize the EUV flux, this stop will also restrict the flow of gas from the source to the monochromator reducing any

contamination issues and the absorption of our EUV wavelengths by that gas.

\subsection{Source stability measurements}

The source has two gas inputs: one for the working gas and a second for a bias gas. The working gas flow is set by the source controller while the operator manually adjusts the flow of the bias gas until there is a stable pressure in the discharge head of $\sim 1 \mathrm{E}^{-3}$ torr. In the measurements shown in the next two sections, the bias and working gas are the same. Each gas that has been used in our source testing has had different bias gas flow rates before the pressure in the lamp and the pulse rate stabilizes. This manual flow adjustment can take as long as 15 minutes for a new gas.

All of the stability measurements used the IRD AXUV-100 photodiode operating in the current mode (see Figures 5 and 10) so that pulses from the source could be captured. Figure 11 shows variations in the pulse height, width and total integrated current. These measurements were made using "ultra-pure" air $\left(\mathrm{N}_{2} / \mathrm{O}_{2}\right.$, see Figure 4). Assuming an "average" wavelength of $15 \mathrm{~nm}$, the quantum efficiency of the IRD AXUV is assumed to be 20 photoelectrons/photon ${ }^{8}$. The number of photons/pulse that the source emitted is shown in Figure 12. This is important in estimating the photons that will be seen by Hi-C and SUVI at the SLF and XRCF (Table 6). This test will be repeated after the monochromator comes online so that we can estimate the photons/pulse at each wavelength band at the entrance to the SUVI and Hi-C telescopes.

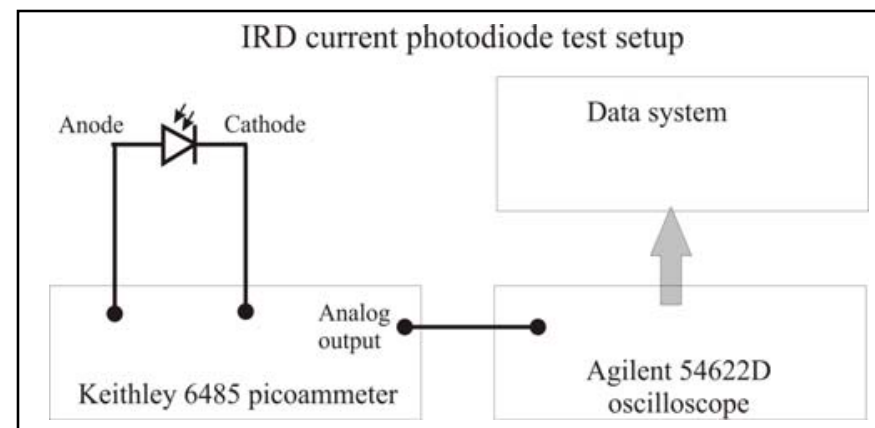

Figure 10. Equipment used to make the current per pulse measurements from the EUV lamp

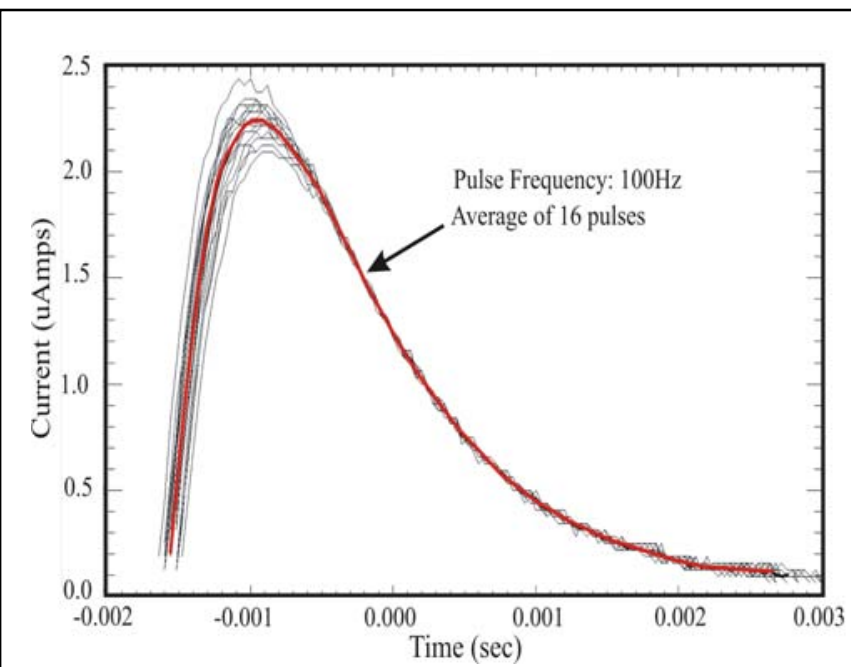

Figure 11. Pulses capture by the CPD during the stability tests. The large red line is the average of 16 pulses captured by our data system.

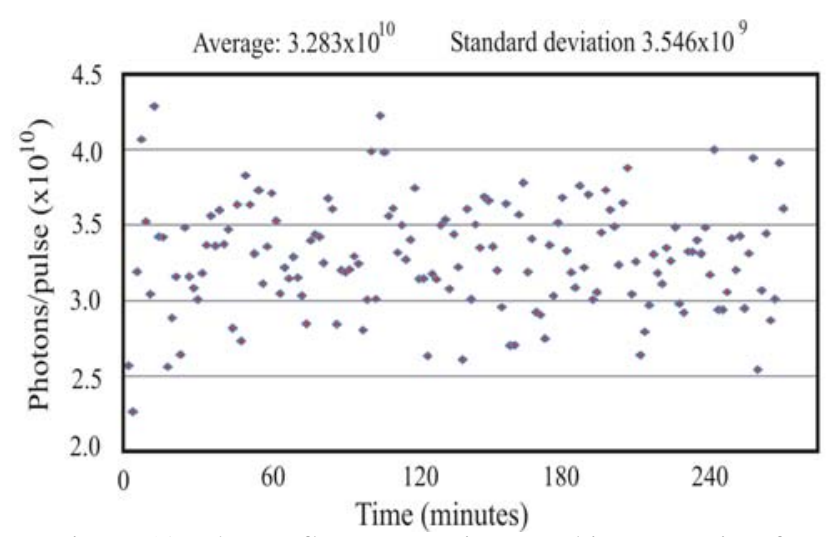

Figure 12. Photon flux versus time . This conversion from $\mu$ Amps to photons assumes $\mathrm{QE}=20$ electrons/photon at average wavelength of $15 \mathrm{~nm}$ (IRD web page ${ }^{7,8}$ ). 


\subsection{Transmission grating characteristics}

As discussed in $§ 4.4$, our initial set of transmission gratings did not have the optical efficiency that we require for monitoring all of the SUVI wavelengths and will be replaced in future upgrades. Although not efficient, measurements were made using the gratings to develop the control and data analysis software and to obtain experience with setting up and aligning these devices. Figure 14 shows the $\mathrm{N} 2 / \mathrm{O}_{2}$ spectra that was observed with only the transmission grating (upper image in the two panels, solid line in the log plot of the intensity/ADU) and with the Luxel Silicon filter inserted into the beam. All of the images are from the same data set and have a logarithmic scale; the only difference is the maximum log scale and the color tables that were used. The full length of the spectrum is $>100 \mathrm{~nm}$ which includes the zero order and small portion of the $-1^{\text {st }}$ order. In this data set there is no

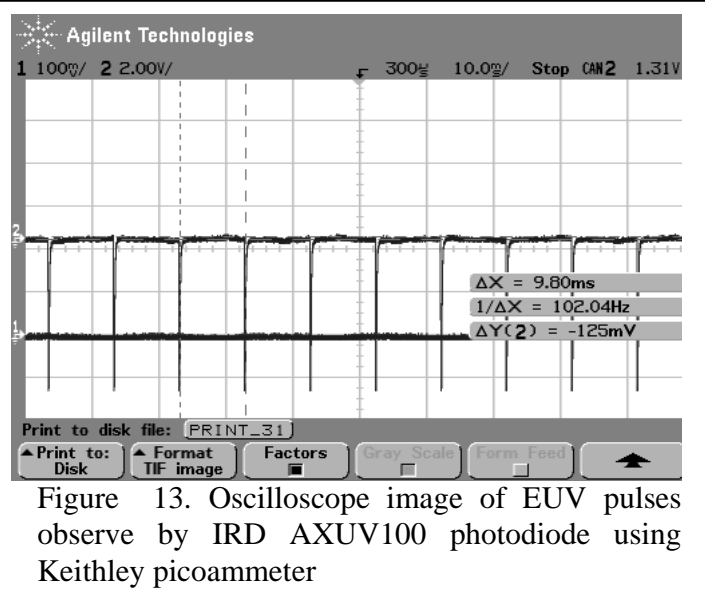
entrance slit so the size of the spectra is set by the aperture of the grating $(1 \mathrm{x} 1 \mathrm{~mm})$. This allowed us to observe weak spectra beyond the SUVI wavelength band. The observations are interesting since the transmission measurements made by NIST (Figure 15) over the SUVI wavelengths implied that the signals above 19nm would be in the noise. Unfortunately, no transmission measurements were made above $30 \mathrm{~nm}$. Therefore, a curve fit of the NIST data assumes that the spectra would follow the exponential decrease that was observed in the $11-17 \mathrm{~nm}$ measurements. Although the signal levels are weak (5second exposure times), it is not clear if the observe spectra is real or $2^{\text {nd }}$ and $3^{\text {rd }}$ order contamination.

Certainly the grating's transmission profile made it difficult to determine the plate scale using the observed spectra. Therefore, a Zemax model of the source, transmission grating and the Andor camera had to be developed. Two models were developed one with the $\mathrm{N} 2 / \mathrm{O}_{2}$ spectrum assuming a perfect grating (no transmission loss as a function of wavelength) and a second model using the NIST data (see Figures 16, 17 and 18). The Zemax model including the NIST transmission data is in good agreement with the observed spectrum shown in Figure 18.

This experiment will be redone when our monochromator comes online and we can control the wavelengths incident on the transmission grating so that we can determine the source of those wavelengths above $20 \mathrm{~nm}$.
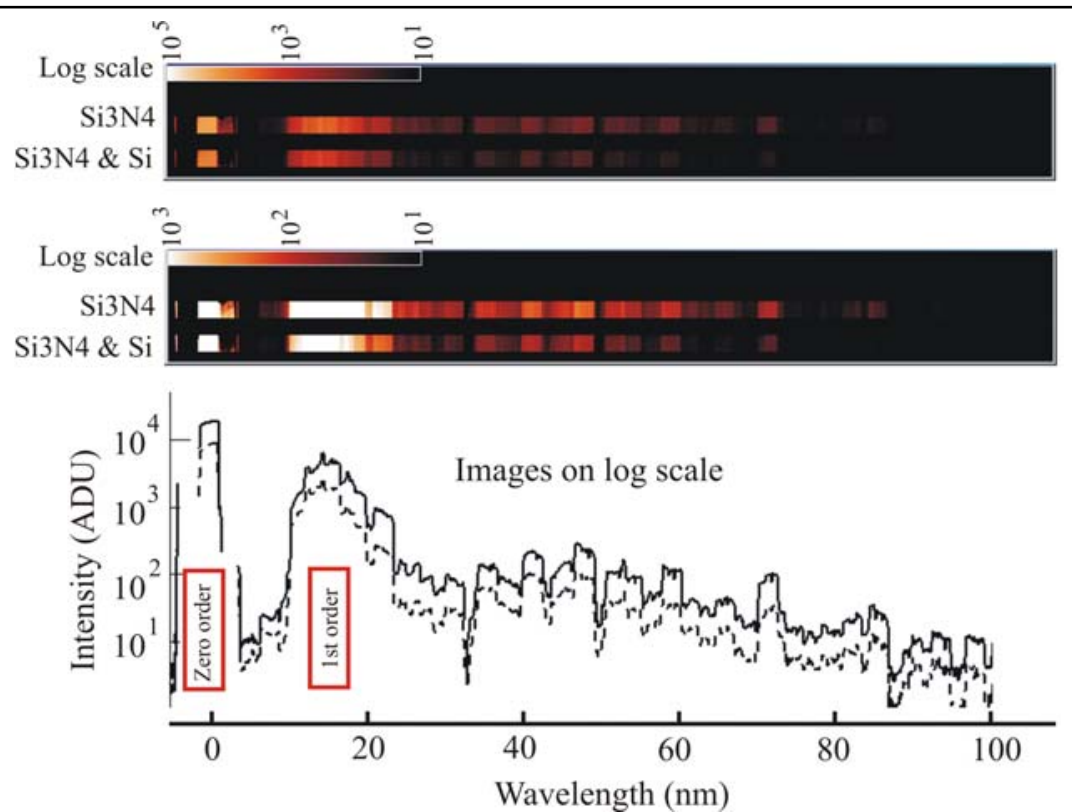

Figure 14. Spectra from our transmission grating on log scales. All images are from the same data set; only the maximum $\log$ scale has been changed. Exposure times for the Andor CCD is 5 seconds. The top image is just the Si3N4 grating (solid line in plot), the lower image (dash line) is with the Luxel Si filter inserted into the beam. 


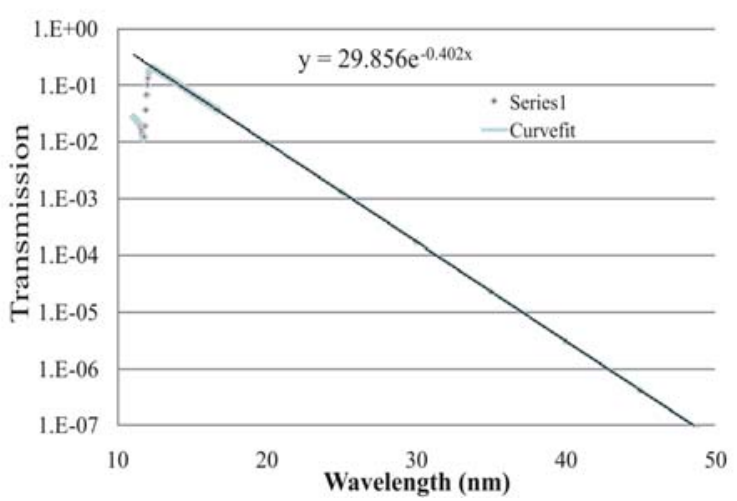

Figure 15. NIST zero order transmission measurements on our gratings $(11-17 \mathrm{~nm})$. An exponential curve fit to the NIST measurements was used to extend the curve to $50 \mathrm{~nm}$.

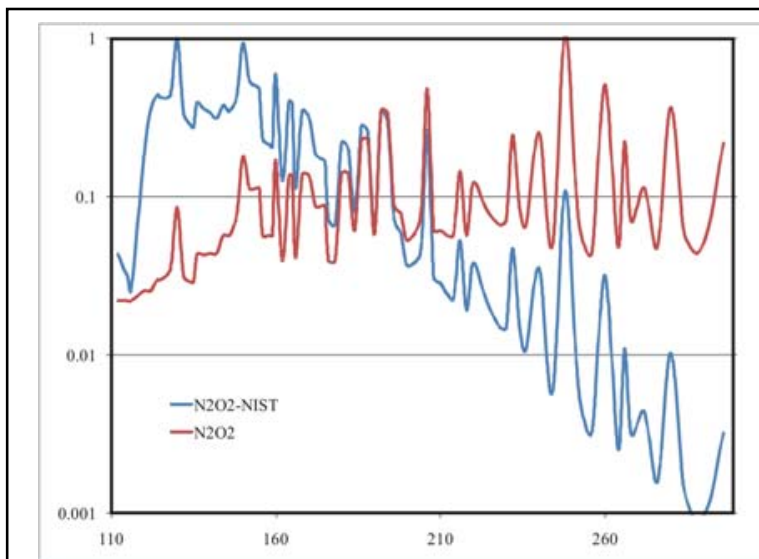

Figure 16. N2/O2 spectrum normalized for input into Zemax wavelength table with weighted strengths normalized to maximum wavelength

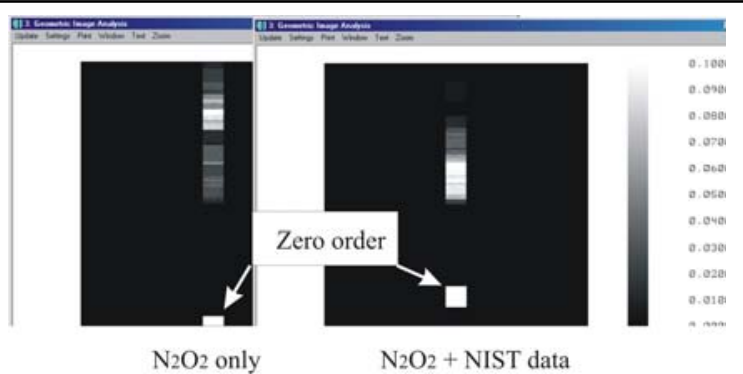

Figure 17. Zemax model of $\mathrm{N} 2 / \mathrm{O}_{2}$ spectrum with and without the NIST transmission data. This shows the inherent problem of determining the plate scale using just the observed spectrum.

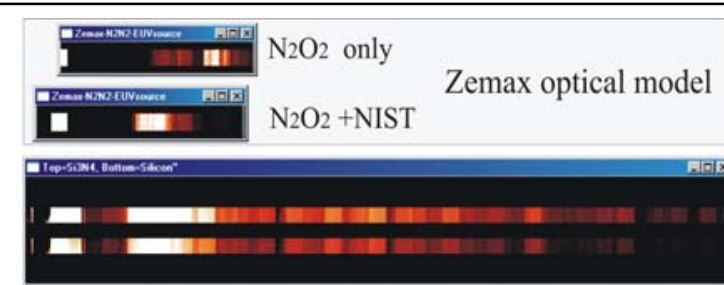

Observed transmission grating spectrum

Figure 18. Zemax model (top images) with transmission grating observations using $\mathrm{N} 2 / \mathrm{O}_{2}$. The top Zemax image assumes a perfect transmission grating with no absorption losses, the bottom image includes the NIST transmission measurements (Figure 15).

Table 6. Photon estimate and beam size at the three MSFC test facilities. The photodiode area is $1 \mathrm{~cm}^{2}$.

\begin{tabular}{|l|c|}
\hline \multicolumn{2}{|c|}{ NSSTC } \\
\hline Photons/ second & $3.3 \times 10^{10}$ \\
\hline Beam size & $1.5 \mathrm{~cm}$ \\
\hline \multicolumn{2}{|c|}{ XRCF } \\
\hline Photons/ second & $9.0 \times 10^{7}$ \\
\hline Beam size & $104 \mathrm{~cm}$ \\
\hline \multicolumn{2}{|c|}{ SLF } \\
\hline Photons/ second & $2.4 \times 10^{9}$ \\
\hline Beam size & $22 \mathrm{~cm}$ \\
\hline
\end{tabular}

Note 1: Two Luxel filters (AL/Si) were inserted into the beam during this test to minimize saturation effects

Note 2: Ultra-pure air used in test. Assumed N2/O2 spectra (Figure 4) and an average QE of 20 photoelectrons/photon ${ }^{8}$. 


\section{SUMMARY}

The gases that we have used with our source are: Xenon, Argon and N2/O2. This Fall we want to: (1) verify that Neon has emission lines in SUVI's 9.4nm wavelength band, (2) have the JY monochromator integrated into our system at the NSSTC, (3) monitor the stability of each of our candidate gases at the SUVI's wavelength bands using this monochromator and (4) vary the high voltage, gas flow and pulse rate to maximize the photon flux at each wavelength band. Also we hope to improve the data transfer rate between the picoammeter and the data system by replacing the Agilent oscilloscope with a USB oscilloscope. Finally, we plan to finish our tests and document the EUV source's performance this Winter so that we can support the Hi-C testing at the SLF by the Spring of 2012.

Finally, we would like to acknowledge Steven Grantham and Charles Tarrio of the National Institute of Standards and Technology (NIST) for their measurements on our transmission gratings and their discussions with us about those measurements.

\section{REFERENCES}

[1] Kegley, J., et.al., “Cryogenic Test Capability at Marshall Space Flight Center's X-ray Cryogenic Facility,” Proc. SPIE 6265, 62654 (2006).

[2] Odell, Stephen, et.al., "X-ray testing Constellation-X optics at MSFC's 100-m facility," Proc. SPIE 5168, 306317 (2004).

[3] http://www.goes-r.gov/org/docs.html

[4] http://www.aixuv.de/DOKUS/PRODUKTINFOS/AIXUV_LAMP_INFOS.pdf

[5] AIXUV user manual

[6] http://www.luxel.com/filter_transmittance.html

[7] http://www.ird-inc.com/

[8] http://www.ird-inc.com/text/axuvopeprin.txt

[9] http://www.goes-r.gov/spacesegment/suvi.html 


\section{MSFC XRCF and SUVI}

MSFC XRCF (X-Ray Calibration Facility) Summary: XRCF operated as a world class $x$-ray and visible optic
test facility for space instrumentation since the 1970 s.

Vacuum tube length: $518 \mathrm{~m}$

Test chamber: $7.3 \mathrm{~m}$ diameter $\mathrm{x} 22.8$ long

Hardware in chamber must meet MSFC 1238 for

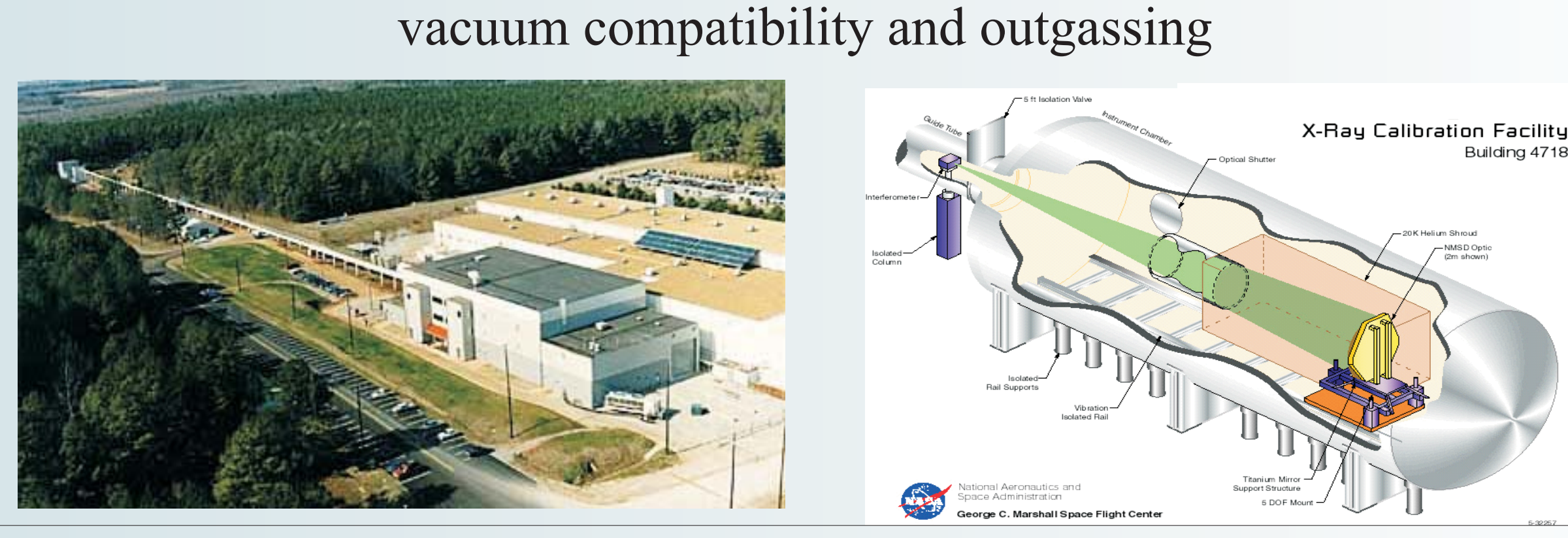

SUVI

Summary:SUVI, the Solar Ultraviolet Imager, is a solar telescope design sk images at high cadence in the extreme ultraviolet (EUV). Combinations of hilters and exposures will allow SUVI emperatures and emission. The purpose is to enable NOAA to monitor solar activity and issue realtime alerts

Requirements on test facility:

- To provide adequate flux levels at SUVI'a six EUV wavelength

- To mon, $17,1,19.5,28.4$ del

cameras for absolute radiometry

- To control source (high voltage and gas flow) to maximize the
flux output at each SUVI wavelength that is being tested

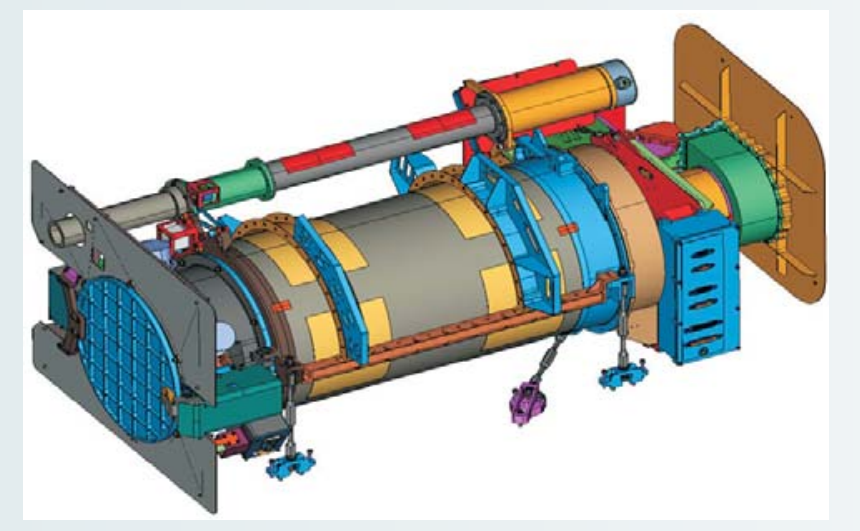

Wavelength vs Gas

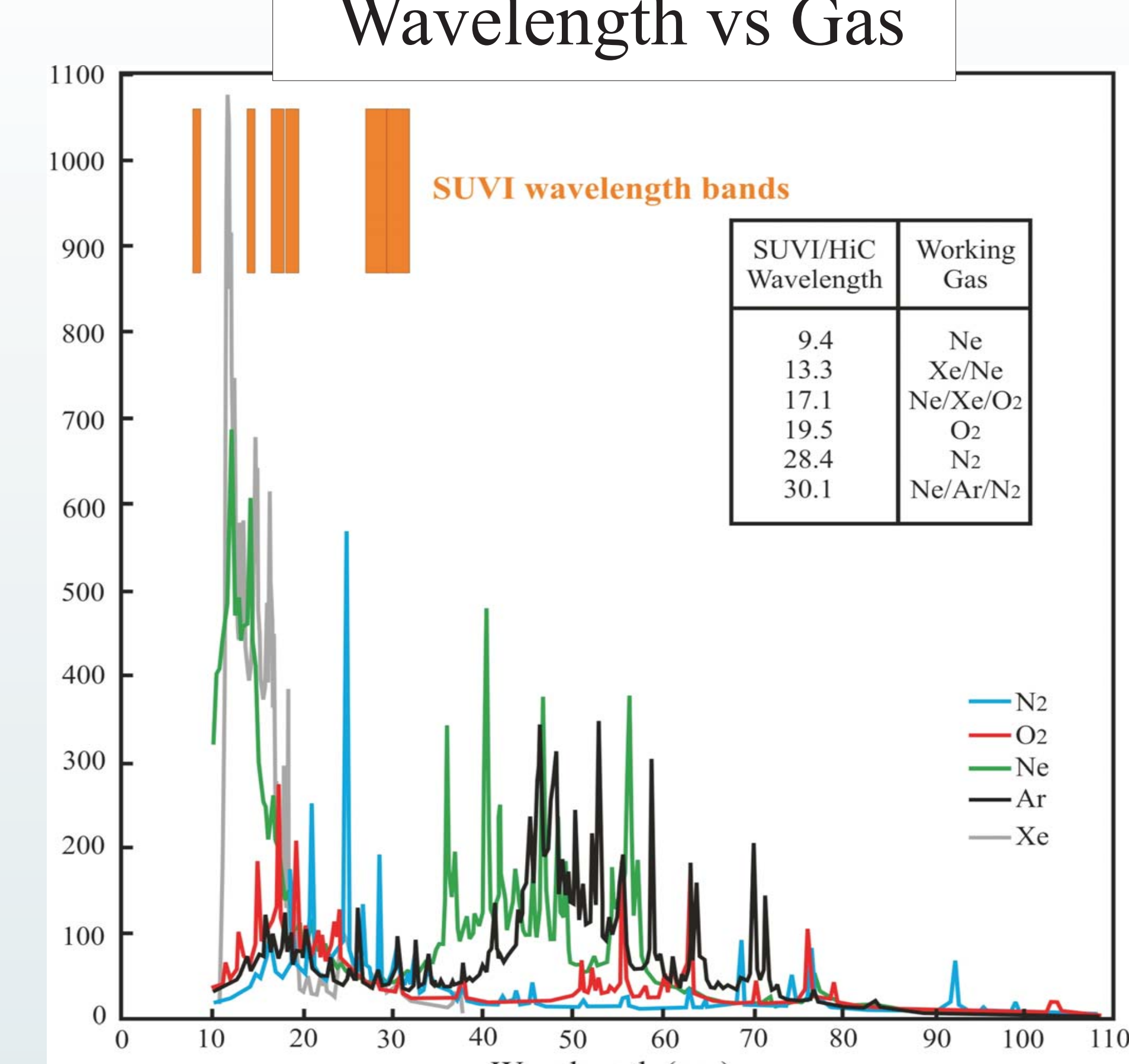

EUV filter characteristics

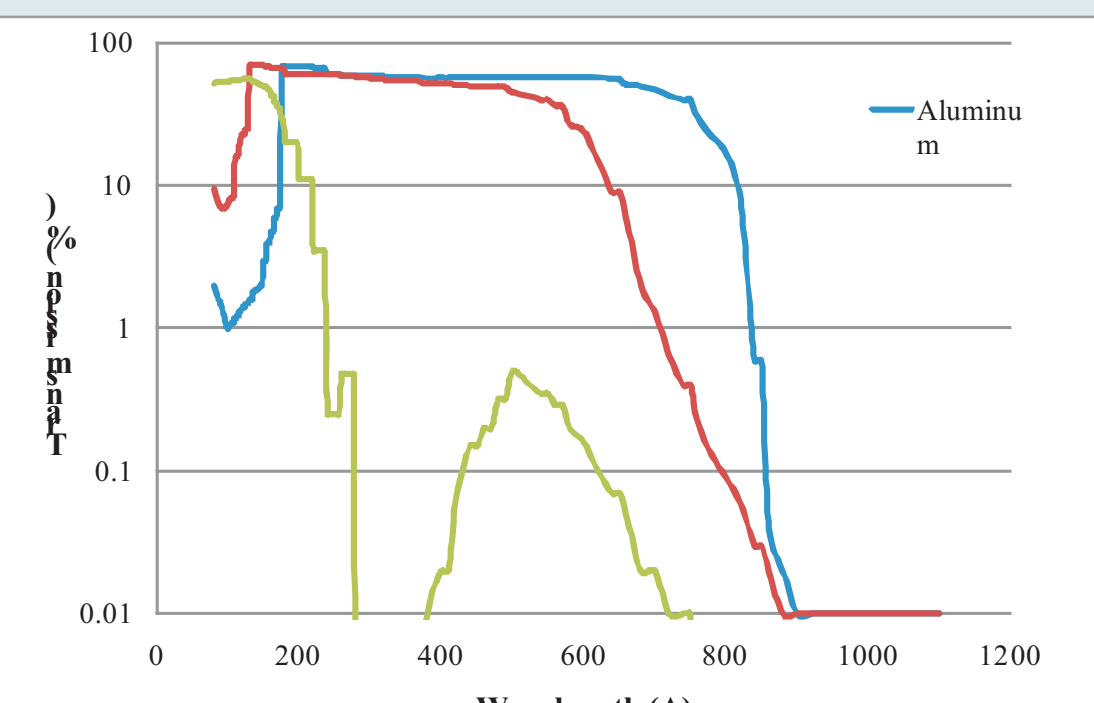

Development of a EUV test facility at the Marshall Space Flight Center

$$
\begin{aligned}
& \begin{array}{l}
\text { Edward West }{ }^{1} \text {, Steve Pavelitz }{ }^{1} \text { Ken Kobayashi }^{2} \text {, Brian Robinson } \\
\text { Jessica Gaskin }{ }^{1} \text {, Amyanthan Cirtain }{ }^{1} \text {, }
\end{array} \\
& \begin{array}{l}
\text { NASA Marshall Space Flight Center, Huntsville AL, } \\
\text { 2 University of Alabama in Huntsville AL, } 3505 \\
3 \text { Miver }
\end{array} \\
& \text { MIT-Lincoln Laboratory, Lexington MA, } 0242 \\
& \text { ABSTRACT }
\end{aligned}
$$

This poster will describe a new EUV test facility that is being developed at the Marshall Space Flight Center to test EUV telescopes. Two flight programs, a high resolution coronal imager (Hil-C, a sounding rocket program) and the Solar Ultraviolet Imager (SUVI, GOES-R telescope, have set the requirement fystems (MSFC Stray Light Facility, MSFC Xray Calibration Facility and the NSSTC ELV test chamber) that will use this facility.

Test chamber:

$+50 \mathrm{~cm}$ dia. $\mathrm{x} 1.9 \mathrm{~m}$ long
+ + Opens to $100 \mathrm{~K}$ clean room

EUV test chamber and detector system
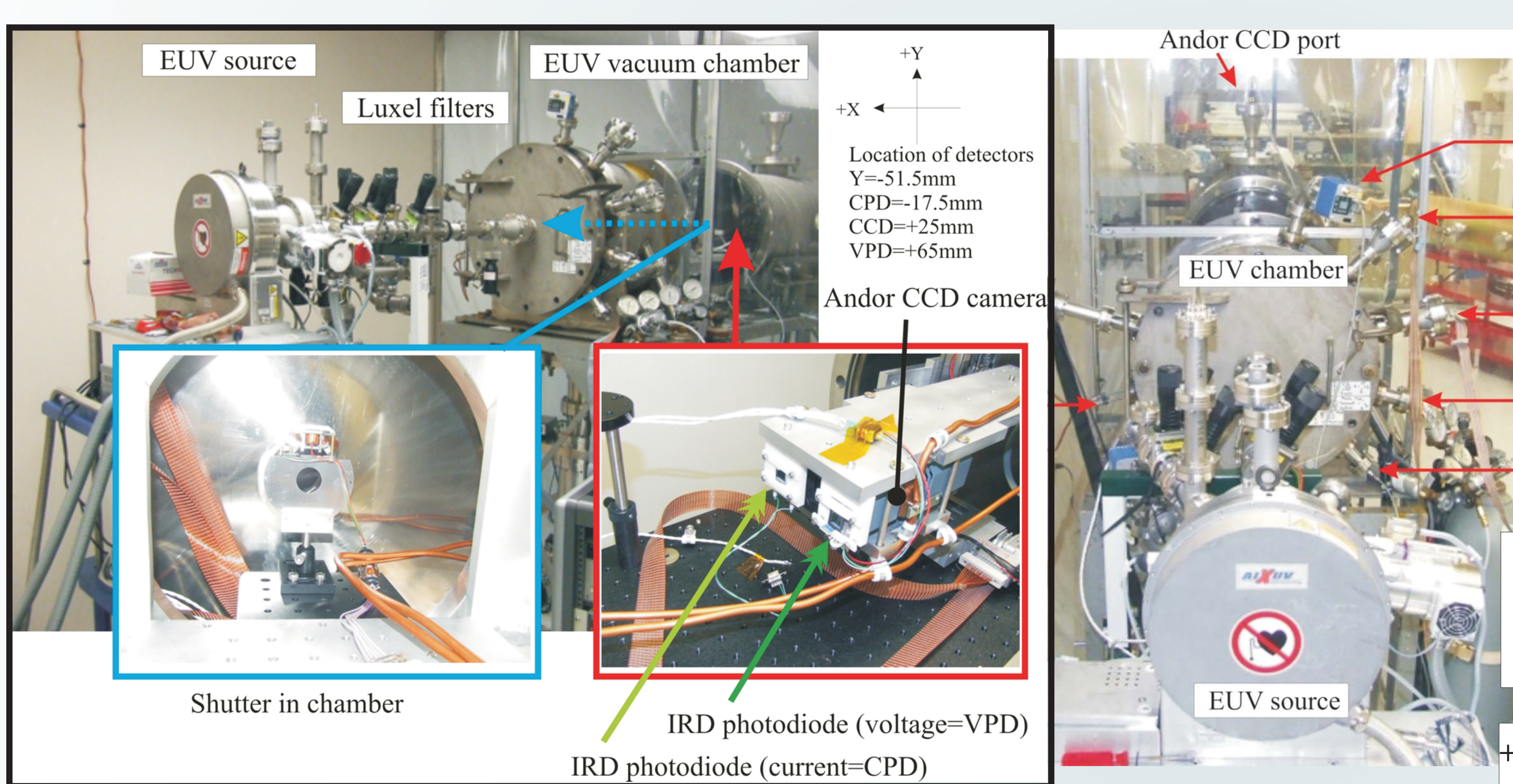

Next phase in test program: JYmonochromator (delivery $\rightarrow 7 / 30 / 2010$

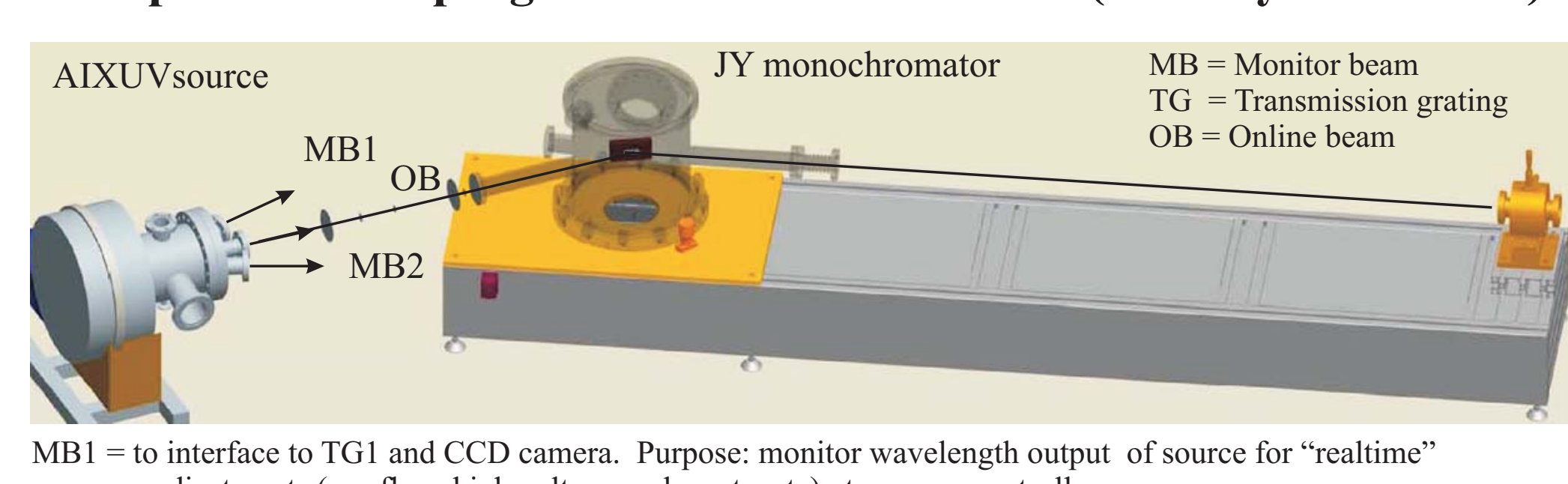

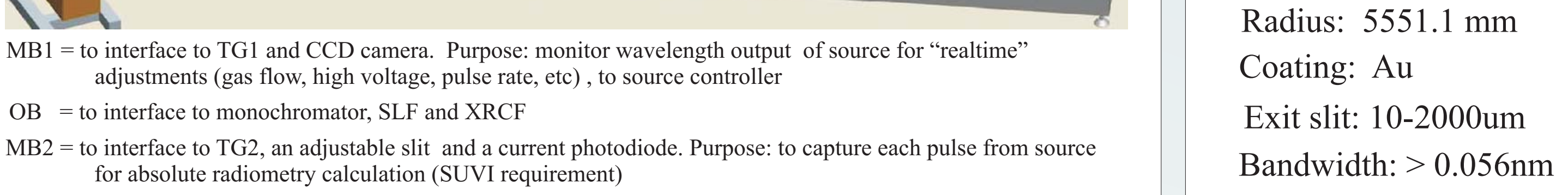

EUV SOURCE CHARACTERISTICS

Photon flux

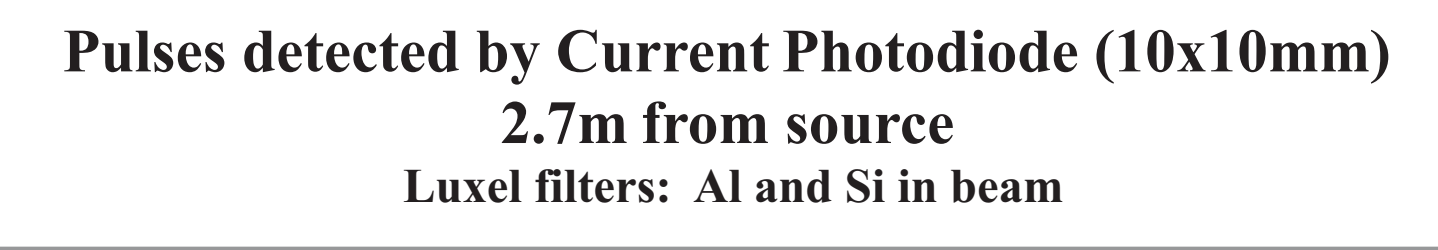

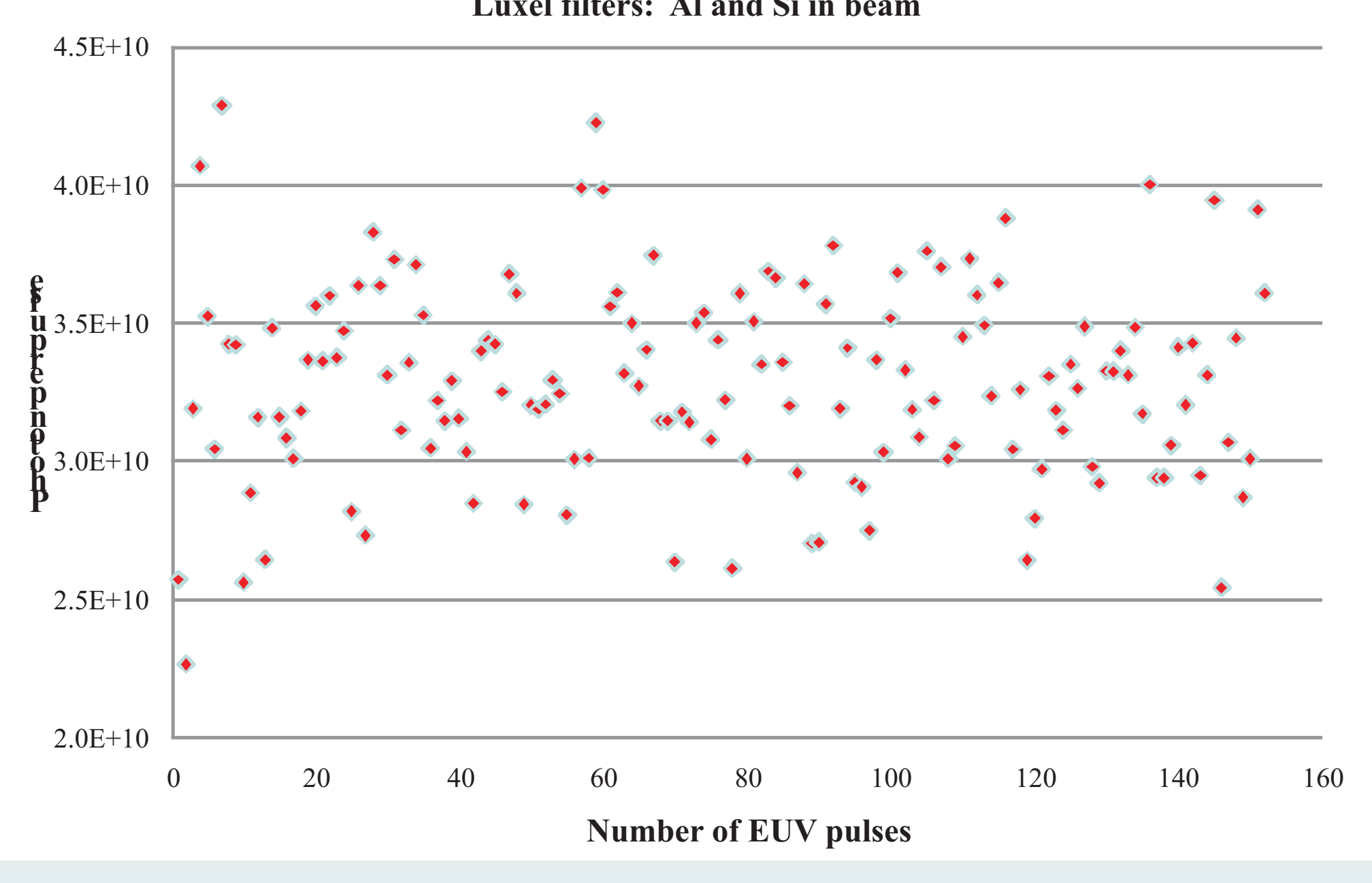

otal current from each pulse, convert to photons on mm
current photodiode (CPD) Assumption:

Average wavelength $=15 \mathrm{~nm}$
IRD AXUV100 QE $=20$

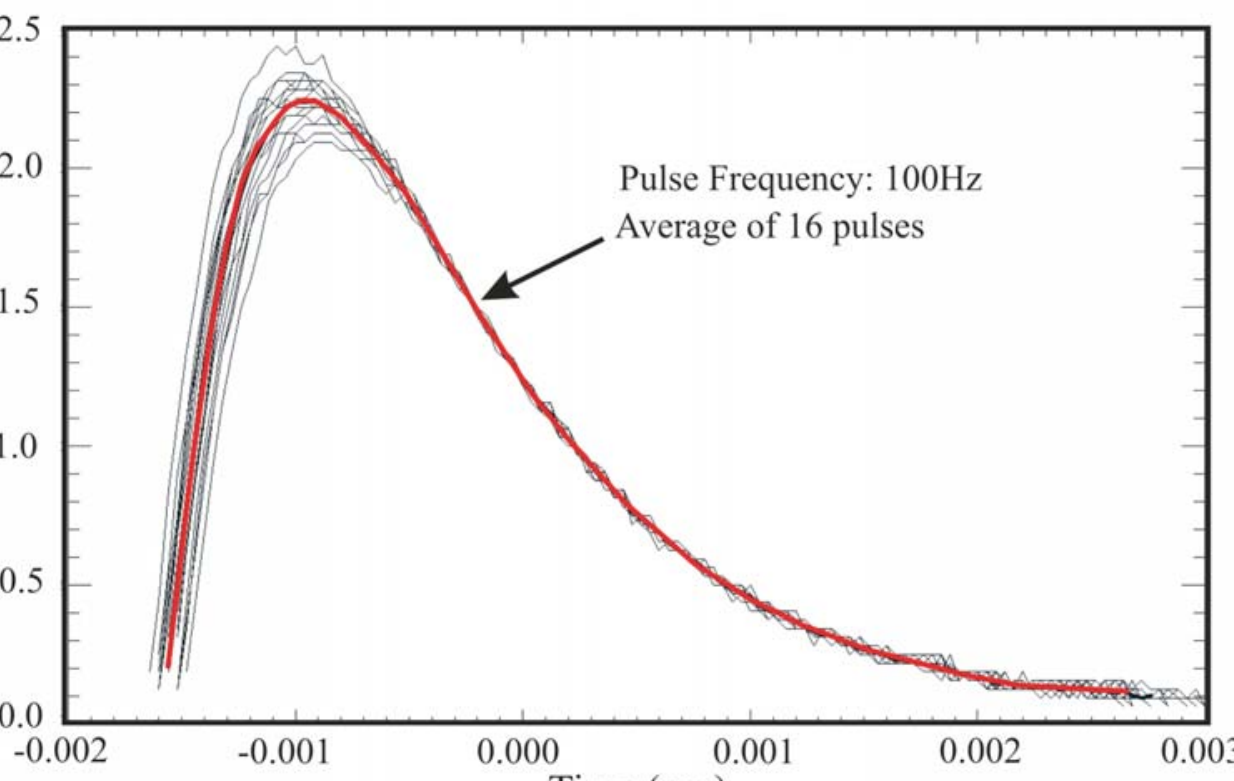

Photon estimated and beam size at:

NSSTC (2.7m) Photons: 3.3 E10 photons/sec (PD 10x10mm) Beam size: $10 \times 10 \mathrm{~cm}$

XRCF (518m) Photons: 9.0E7 photons/sec (PD 10x10mm)

SLF (102m) Photons: 2.4E9 photons/sec (PD 10x10mm) Beam size: $22 \mathrm{~cm}$

NOTE 1: Estimates based on measurements WITH Aluminum and Silicon filters inserted into bean
Number of photons will increase if if these filters not required.

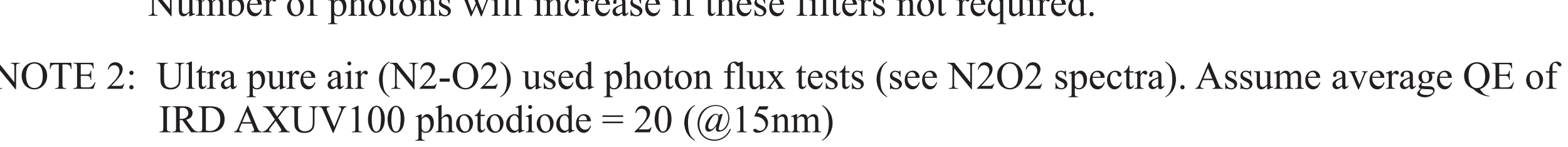

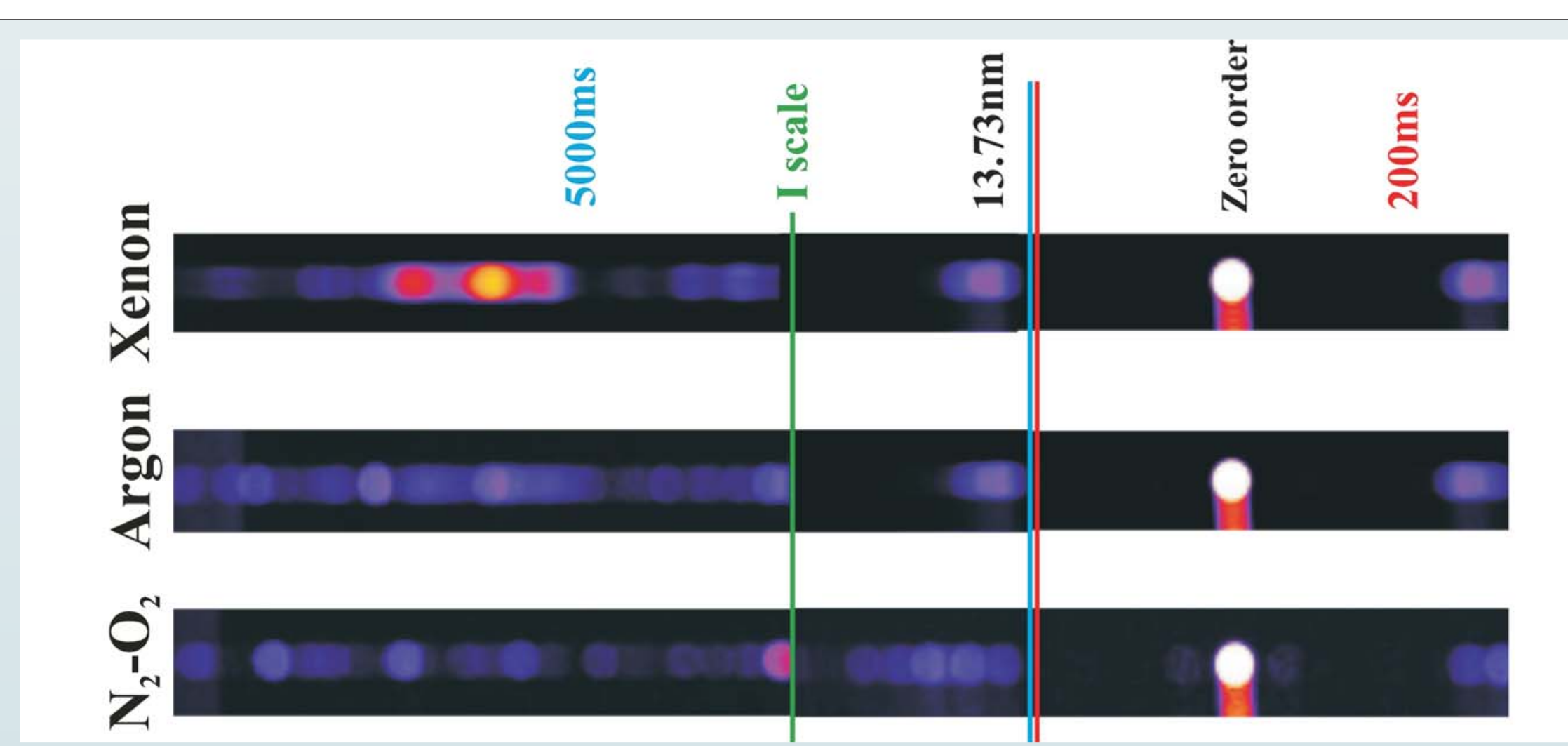

MSFC SLF and HiC

MSFC SLF (Stray Light Facility) SLF originally designed for stray-light testing of visitlo
light space-flight optics. Currently used to evaluate
and calibrate high energy optics and detectors.

Vacuum tube: $1.2 \mathrm{~m}$ diamter $\mathrm{x} 92 \mathrm{~m}$ long

Test chamber: $3 \mathrm{~m}$ diameter $\mathrm{x} 10 \mathrm{~m}$ long Opens to $10 \mathrm{~K}$ clean room Hardware in chamber must meet MSFC 1238 for vacuum compatibility and outgassing

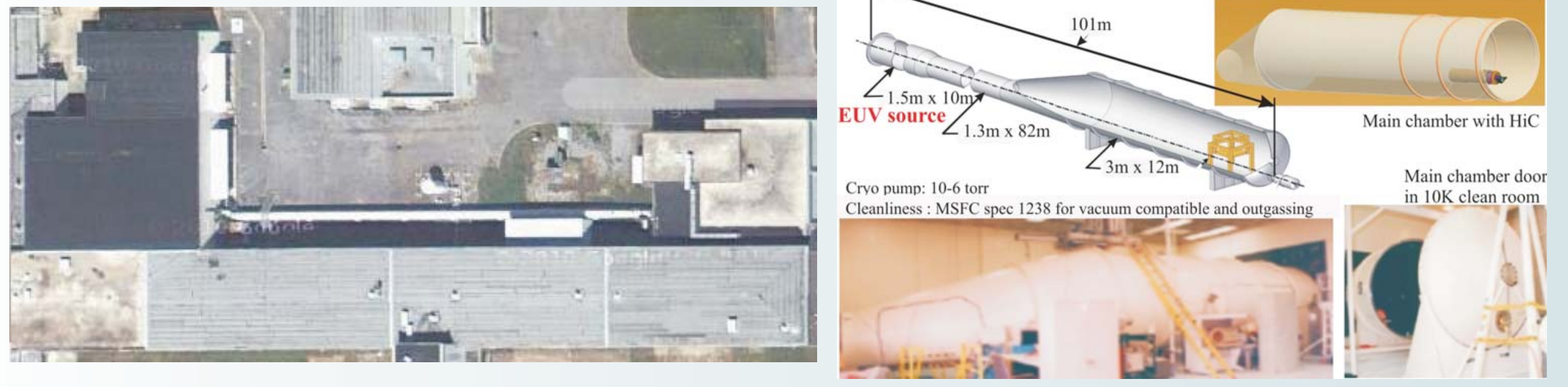

$\mathrm{HiC}$

Summary: HiC, the High Resolution Coronal Imager, is the first telescope designed to take high resolution $(170 \mathrm{~km})$ images
the extreme ultraviolet The intent of this sounding rocket in the extreme ultraviolet. The intent of this sounding rocket
program is to demonstrate the technology to acquire images with this resolution and investigate the scientific value of these images. HiC is a Ritchey-Chrietien telescope with
super-polished mirrors that will provide 0.1 arcsec pixels. super-polished mirrors that will provide 0.1 arcsec pixels.
The mirrors have a multilayer coating that will reflect the target wavelength $(19.5 \mathrm{~nm})$ to a large format $\mathrm{CCD}$ camera.

Requirements on test facility:

adequate flux levels at $19.5 \mathrm{~nm}$ at the SLF

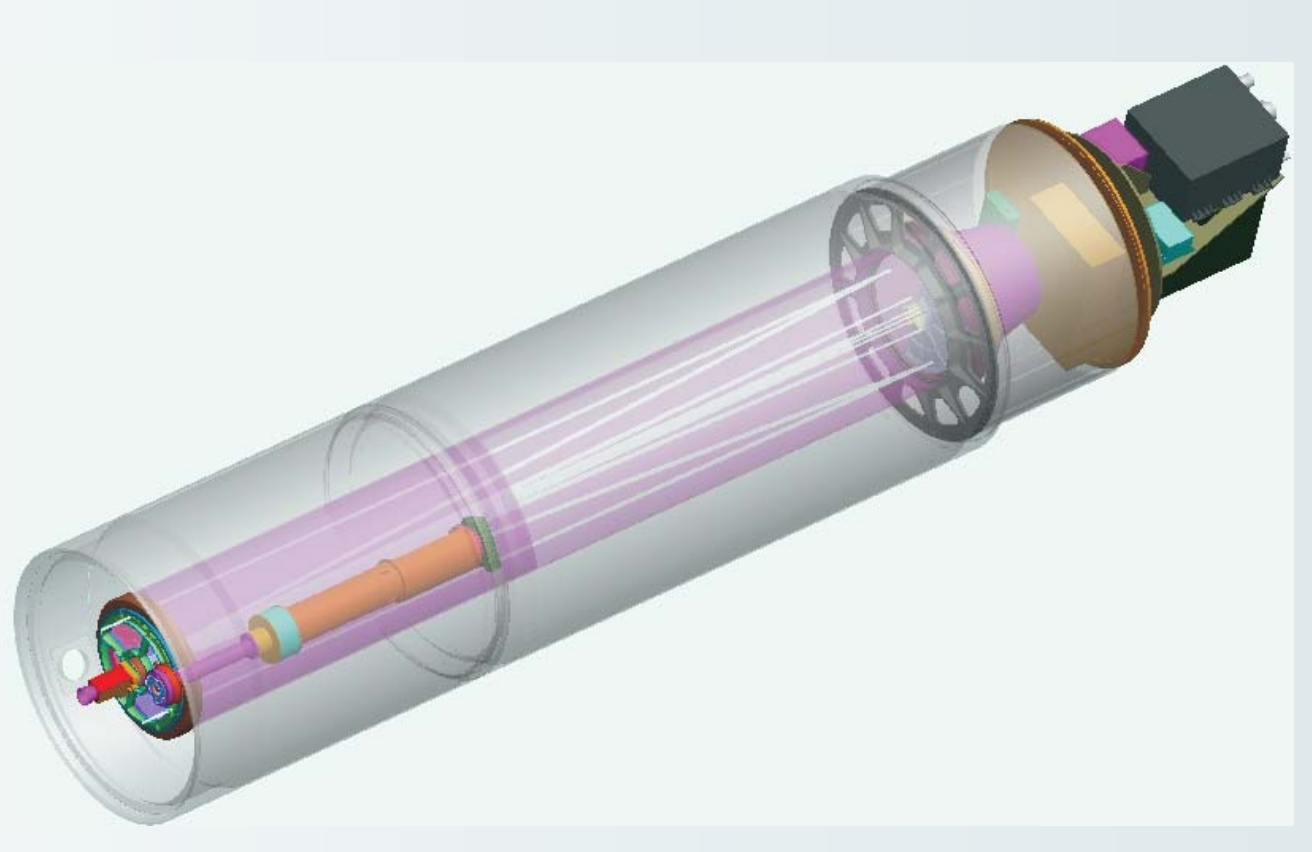

Transmission Gratings (TG1 and TG2) pose: To be monitor the wavelength characteristics of the source. MB1: TG1 and the CCD camera to observe a large wavelength band
MB2: TG2 and the current photodiode for a narrow wavelength band and absolute radiometry measurem ransmission properties of TC
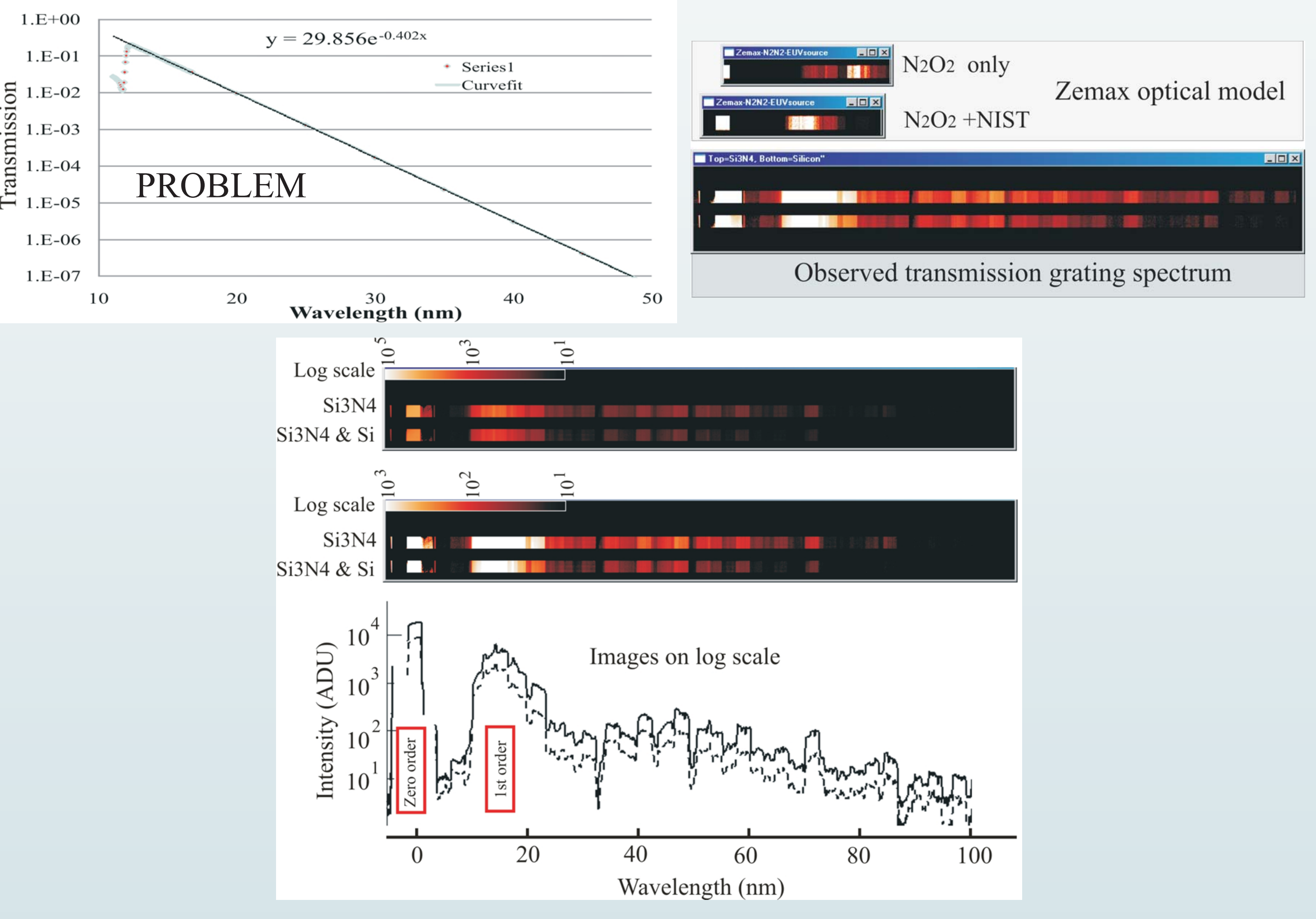